Supporting Information for:

\title{
Highly Enantiomerically Enriched Chlorophosphine Boranes: Synthesis and Applications as P-Chirogenic Electrophilic blocks
}

Christophe Bauduin, ${ }^{b}$ Dominique Moulin, ${ }^{b}$ El Bachir Kaloun, ${ }^{b}$ Christophe Darcela and Sylvain Jugé*a

a Laboratoire de Synthèse et d'Electrosynthèse Organométalliques, UMR 5632,

Université de Bourgogne, 6 Bd Gabriel, BP 138, 21100 Dijon, France;

Fax:33103803960 98; e-mail:Sylvain.Juge@u-Bourgogne.fr

b Université de Cergy Pontoise, 5 mail Gay Lussac, 95031 Cergy Pontoise, France

Table of contents: Synthesis of the aminophosphine boranes 11a-h. Structures and crystal datas for the $(S \mathrm{p})-(+)-\mathrm{N}$-methyl[(1R,2S)(2-hydroxy-1-phenyl)ethyl]aminotert-butylphenylphosphine borane $\mathbf{1 1 h},(R)$-o-anisylphenyl-o-tolylphosphine 14, $(S, S)$-()-1,2-bis(methylphenyl phosphinito borane)benzene 18, and for the $(+)$-N,N'-bis $[(R)-o$ anisylphenylphosphino borane] ethylene diamine $\mathbf{2 0}$.

\section{Preparation of aminophosphine boranes $11 \mathrm{a}-\mathrm{h}$.}

Preparation of aryllithium reagents by metal-halide exchange: In a two necked-flask equipped with a magnetic stirrer, 1 equiv of sec-butyllithium is added. The mixture is allowed to cool to $0^{\circ} \mathrm{C}$ and 1 equiv of 1-bromoanisol (1-bromonaphthalene or bromobenzene) is slowly added with a syringe while stirring. After formation of a white precipitate, the mixture is stirred for $1 \mathrm{~h}$ at $0^{\circ} \mathrm{C}$. The organolithium reagent is dissolved with a minimum of dry THF before use.

In the case of the solid 2-bromonaphthalene, 1 equiv of aryl halide is introduced in a flask, and 1 equiv of sec-butyllithium is slowly added with a syringe while stirring at $0^{\circ} \mathrm{C}$. The rest of the procedure is similar to that described above. 
Preparation of the cyclohexyllithium reagent from cyclohexyl chloride: In a three necked flask, equipped with a condenser and an addition funnel, under a dry atmosphere, $50 \mathrm{~mL}$ of petroleum ether $\left(40-60^{\circ} \mathrm{C}\right)$ and metallic lithium (cut in small portions) (1.74 $\mathrm{g}$, 5 equiv) were added. A solution of cyclohexyl chloride $(5.9 \mathrm{~mL}, 1$ equiv) in $10 \mathrm{~mL}$ of petroleum ether were added dropwise over a $4 \mathrm{~h}$ period under a vigourous stirring. The reaction was started by warming with a hot water bath. At the end of the addition, the reflux was continued for one more hour. The mixture reaction changed to a violet color. After filtration through a bed of Celite, the collected yellow solution was used without further purification and was titrated using classical techniques.

General procedure. In a $100 \mathrm{~mL}$ three-necked flask, equipped with a magnetic stirrer and an argon inlet, $15 \mathrm{mmol}$ of the oxazaphospholidine borane complex $\mathbf{1 0}$ were dissolved in $15 \mathrm{~mL}$ of anhydrous THF. The mixture was cooled at $-78^{\circ} \mathrm{C}$ and 2 equiv (30 mmol) of the organolithium reagent previously prepared were slowly added. The resulting mixture was stirred and warmed to $0^{\circ} \mathrm{C}$ until the starting material had completely reacted. The reaction was monitored by TLC over silica (toluene as eluent), and was finally hydrolyzed at $0^{\circ} \mathrm{C}$ with $5 \mathrm{~mL}$ of water. The $\mathrm{THF}$ was removed under reduced pressure and the aqueous layer was extracted several times with dichloromethane. The combinated organic phases were dried over magnesium sulfate and the solvent was removed. The residue was purified on a short column of silica gel, using a mixture of toluene/AcOEt 95:5 as eluent, to afford the aminophosphine boranes 11 in excellent yields of up to $95 \%$. The aminophosphine boranes can be recrystallized using a mixture hexane/isopropanol 7:3.

\section{$\left(S_{\mathrm{P}}\right)-(+)-\mathrm{N}-$ Methyl-N-[(1S,2R)(1-hydroxy-1-phenyl-prop-2-yl)] aminomethyl}

phenylphosphine borane 11a.18a,20 Yield $=95 \% ;$ White cystals: $\mathrm{mp}=67^{\circ} \mathrm{C}$ $(i \mathrm{PrOH} / \mathrm{Hexane} 1: 1) ; R_{\mathrm{f}}=0.45($ toluene/AcOEt $9: 1) ;[\alpha]_{\mathrm{D}}{ }^{25}=+12.8\left(\mathrm{c} 1.0, \mathrm{CH}_{2} \mathrm{Cl}_{2}\right)$; IR $\left(\mathrm{KBr}, v \mathrm{~cm}^{-1}\right): 3354,3057-2873,2376,1437,1136,1116,1066,1049,906$; 
${ }^{1} \mathrm{H} \mathrm{NMR}\left(\mathrm{CDCl}_{3}\right): \delta(\mathrm{ppm}) 0.00-1.60(3 \mathrm{H}, \mathrm{br}), 1.22\left(3 \mathrm{H}, \mathrm{d},{ }^{3} J_{\mathrm{H}-\mathrm{H}}=6.7\right), 1.50(3 \mathrm{H}, \mathrm{d}$, $\left.{ }^{2} J_{\mathrm{P}-\mathrm{H}}=9.0\right), 2.15(1 \mathrm{H}, \mathrm{br}), 2.45\left(3 \mathrm{H}, \mathrm{d},{ }^{3} J_{\mathrm{P}-\mathrm{H}}=8.6\right), 4.00(1 \mathrm{H}, \mathrm{m}), 4.70\left(1 \mathrm{H}, \mathrm{d},{ }^{3} J_{\mathrm{H}-\mathrm{H}}=\right.$ 6.3), 7.04-7.11 $(3 \mathrm{H}, \mathrm{m}), 7.21-7.42(7 \mathrm{H}, \mathrm{m}) ;{ }^{13} \mathrm{C} \mathrm{NMR}\left(\mathrm{CDCl}_{3}\right): \delta(\mathrm{ppm}) 11.2\left(\mathrm{~d},{ }^{1} J_{\mathrm{P}-\mathrm{C}}=\right.$ $41.7), 13.8,28.9,59.0\left(\mathrm{~d},{ }^{2} J_{\mathrm{P}-\mathrm{C}}=7.6\right), 77.6\left(\mathrm{~d},{ }^{3} J_{\mathrm{P}-\mathrm{C}}=5.9\right), 126.7,128.1\left(\mathrm{~d}, J_{\mathrm{P}-\mathrm{C}}=\right.$ $27.7), 128.4,128.5,130.2\left(\mathrm{~d}, J_{\mathrm{P}-\mathrm{C}}=10.0\right), 130.5\left(\mathrm{~d}, J_{\mathrm{P}-\mathrm{C}}=2.3\right), 132.8\left(\mathrm{~d}, J_{\mathrm{P}-\mathrm{C}}=65.4\right)$, $142.6 ;{ }^{31} \mathrm{P}$ NMR $\left(\mathrm{CDCl}_{3}\right): \delta(\mathrm{ppm})+66.5\left(\mathrm{q},{ }^{1} J_{\mathrm{P}-\mathrm{B}}=83\right) ; \mathrm{MS}(\mathrm{EI}, 70 \mathrm{eV}): \mathrm{m} / \mathrm{z} 272\left(\mathrm{M}^{+}-\right.$ $\left.\mathrm{BH}_{3}-\mathrm{CH}_{3} ; 1\right), 180$ (100), 132 (21), 155 (54), 123 (54); Anal. calcd for $\mathrm{C}_{17} \mathrm{H}_{25} \mathrm{BNOP}$ (301.1713): C 67.80, H 8.37, N 4.65; Found: C 67.75, H 8.56, N 4.64

\section{$(R p)-(-)-N-M e t h y l[(1 R, 2 S)(2-h y d r o x y-1-p h e n y l)$ ethyl $]$ amino-o-anisylphenyl}

phosphine borane 11b. ${ }^{20}$ Yield $=95 \%$; White crystals $(i \mathrm{PrOH} /$ Hexane $) ; \mathrm{mp}=111-$ $112^{\circ} \mathrm{C} ; \mathrm{R}_{\mathrm{f}}=0.45($ toluene/AcOEt $9: 1) ;[\alpha]_{\mathrm{D}}{ }^{25}=-38.3\left(\mathrm{c} 1.0, \mathrm{CH}_{2} \mathrm{Cl}_{2}\right) ; \mathrm{IR}\left(\mathrm{KBr}, \mathrm{v} \mathrm{cm}{ }^{-1}\right)$ : $3500,2950-2900,2381,1589,1574,1477,1462,1431,1276,1251,1044 ;{ }^{1} \mathrm{H}$ NMR $\left(\mathrm{CDCl}_{3}\right): \delta(\mathrm{ppm})$ 0.20-2.00 $(3 \mathrm{H}, \mathrm{sl}), 1.20\left(3 \mathrm{H}, \mathrm{d},{ }^{3} J_{\mathrm{H}-\mathrm{H}}=7.8\right), 2.09(1 \mathrm{H}, \mathrm{s} 1), 2.52(3 \mathrm{H}$, $\left.\mathrm{d},{ }^{3} J_{\mathrm{P}-\mathrm{H}}=8.1\right), 3.53(3 \mathrm{H}, \mathrm{s}), 4.31(1 \mathrm{H}, \mathrm{m}), 4.85\left(1 \mathrm{H}, \mathrm{d},{ }^{3} J_{\mathrm{H}-\mathrm{H}}=5.6\right), 6.86-6.91(1 \mathrm{H}, \mathrm{m})$, 6.96-7.02 (1H, m), 7.02-7.15 (1H, m), 7.15-7.36 (7H, m), 7.36-7.45 (3H, m), 7.45-7.60 $(1 \mathrm{H}, \mathrm{m}) ;{ }^{13} \mathrm{C} \mathrm{NMR}\left(\mathrm{CDCl}_{3}\right): \delta(\mathrm{ppm}) 12.5\left(\mathrm{~d}, J_{\mathrm{P}-\mathrm{C}}=2.1\right), 30.9\left(\mathrm{~d}, J_{\mathrm{P}-\mathrm{C}}=3.8\right), 55.0,58.1$ $\left(\mathrm{d},{ }^{2} J_{\mathrm{P}-\mathrm{C}}=10.4\right), 78.8\left(\mathrm{~d},{ }^{3} J_{\mathrm{P}-\mathrm{C}}=5.0\right), 111.5\left(\mathrm{~d}, J_{\mathrm{P}-\mathrm{C}}=4.7\right), 118.5\left(\mathrm{~d}, J_{\mathrm{P}-\mathrm{C}}=56.9\right), 120.8$ $\left(\mathrm{d}, J_{\mathrm{P}-\mathrm{C}}=10.5\right), 126.5,127.7\left(\mathrm{~d}, J_{\mathrm{P}-\mathrm{C}}=16.9\right), 128.0,128.3,129.9\left(\mathrm{~d}, J_{\mathrm{P}-\mathrm{C}}=2.3\right), 130.8$ $\left(\mathrm{d}, J_{\mathrm{P}-\mathrm{C}}=10.4\right), 132.2\left(\mathrm{~d}, J_{\mathrm{P}-\mathrm{C}}=71.3\right), 133.2\left(\mathrm{~d}, J_{\mathrm{P}-\mathrm{C}}=1.6\right), 134.9\left(\mathrm{~d}, J_{\mathrm{P}-\mathrm{C}}=10.8\right)$, $142.5,161.0\left(\mathrm{~d}, J_{\mathrm{P}-\mathrm{C}}=2.5\right) ;{ }^{31} \mathrm{P} \mathrm{NMR}\left(\mathrm{CDCl}_{3}\right): \delta(\mathrm{ppm})+69.5(\mathrm{br}) ; \mathrm{MS}(\mathrm{EI}, 70 \mathrm{eV})$ : m/z $286\left(\mathrm{M}^{+}-o \mathrm{An} ; 13\right), 272\left(\mathrm{M}^{+}-o \mathrm{An}-\mathrm{BH}_{3} ; 53\right), 232$ (37), 216 (65), 146 (50), 91 (93), 58 (100); Anal. calcd for $\mathrm{C}_{23} \mathrm{H}_{29} \mathrm{BNO}_{2} \mathrm{P}$ (393.2667): C 70.24, H 7.43, N 3.56; Found: C $70.12, \mathrm{H} 7.15, \mathrm{~N} 3.50$. 
( Rp) - ( - ) -N-Methyl [ ( $1 R, 2 S)(2-h y d \operatorname{roxy}-1$-phenyl ) ethyl ] aminophenyl-o-tolyl

phosphine borane 11c. Yield $=92 \%$; White crystals $(i \mathrm{PrOH} /$ Hexane $) ; \quad \mathrm{R}_{\mathrm{f}}=0.40$ (toluene/AcOEt 9:1); $\mathrm{mp}=105^{\circ} \mathrm{C} ;[\alpha]_{\mathrm{D}}{ }^{25}=-76.0\left(\mathrm{c} 2.0, \mathrm{CH}_{2} \mathrm{Cl}_{2}\right) ; \mathrm{IR}\left(\mathrm{KBr}, \mathrm{v} \mathrm{cm}{ }^{-1}\right)$ : 3613, 2979, 2403, 1588, 1455, 1434; ${ }^{1} \mathrm{H} \mathrm{NMR}\left(\mathrm{CDCl}_{3}\right): \delta(\mathrm{ppm})$ 0.20-2.00 (3H, br), $1.17\left(3 \mathrm{H}, \mathrm{d},{ }^{3} J_{\mathrm{H}-\mathrm{H}}=6.9\right), 2.24(3 \mathrm{H}, \mathrm{s}), 2.64\left(3 \mathrm{H}, \mathrm{d},{ }^{3} J_{\mathrm{P}-\mathrm{H}}=8.0\right), 4.38(1 \mathrm{H}, \mathrm{m}), 4.96(1 \mathrm{H}$, $\left.\mathrm{d},{ }^{3} J_{\mathrm{H}-\mathrm{H}}=4.0\right), 7.10-7.45(11 \mathrm{H}, \mathrm{m}), 7.49-7.65(3 \mathrm{H}, \mathrm{m}) ;{ }^{13} \mathrm{C} \mathrm{NMR}\left(\mathrm{CDCl}_{3}\right): \delta(\mathrm{ppm}) 11.4$ $\left(\mathrm{d}, J_{\mathrm{P}-\mathrm{C}}=4.6\right), 22.0\left(\mathrm{~d}, J_{\mathrm{P}-\mathrm{C}}=3.7\right), 31.3\left(\mathrm{~d}, J_{\mathrm{P}-\mathrm{C}}=3.6\right), 58.0\left(\mathrm{~d},{ }^{2} J_{\mathrm{P}-\mathrm{C}}=10.3\right), 79.0(\mathrm{~d}$, $\left.{ }^{3} J_{\mathrm{P}-\mathrm{C}}=2.0\right), 125.6\left(\mathrm{~d}, J_{\mathrm{P}-\mathrm{C}}=9.5\right), 125.9,127.4,128.3,128.4\left(\mathrm{~d}, J_{\mathrm{P}-\mathrm{C}}=10.0\right), 128.7(\mathrm{~d}$, $\left.J_{\mathrm{P}-\mathrm{C}}=64.1\right), 130.8\left(\mathrm{~d}, J_{\mathrm{P}-\mathrm{C}}=2.1\right), 130.9\left(\mathrm{~d}, J_{\mathrm{P}-\mathrm{C}}=2.3\right), 131.9\left(\mathrm{~d}, J_{\mathrm{P}-\mathrm{C}}=9.9\right), 132.1(\mathrm{~d}$, $\left.J_{\mathrm{P}-\mathrm{C}}=60.9\right), 132.2\left(\mathrm{~d}, J_{\mathrm{P}-\mathrm{C}}=9.2\right), 132.7\left(\mathrm{~d}, J_{\mathrm{P}-\mathrm{C}}=8.1\right), 142.2\left(\mathrm{~d}, J_{\mathrm{P}-\mathrm{C}}=12.2\right), 142.5$; ${ }^{31} \mathrm{P}$ NMR $\left(\mathrm{CDCl}_{3}\right): \delta(\mathrm{ppm})+71.5(\mathrm{br}) ; \mathrm{MS}(\mathrm{EI}, 70 \mathrm{eV}): \mathrm{m} / \mathrm{z} 376\left(\mathrm{M}^{+}-\mathrm{H} ; 20\right), 270(10)$; 256 (60), 216 (15), 199 (60), 148 (35), 91 (27), 58 (100); Anal. calcd for $\mathrm{C}_{23} \mathrm{H}_{29} \mathrm{BNOP}$ (377.2673): C 73.22, H 7.75; Found: C 73.10, H 7.73.

\section{$(R p)-(-)-N-M e t h y l[(1 R, 2 S)(2-h y d$ roxy-1-phenyl) ethyl $]$-amino-1-naphthylphenyl}

phosphine borane 11d. 31 Yield $=87 \%$; White crystals $(i \mathrm{PrOH} / \mathrm{Hexane}) ; \mathrm{R}_{\mathrm{f}}=0.55$ (toluene/AcOEt 9:1); $\mathrm{mp}=112-113^{\circ} \mathrm{C} ;[\alpha]_{\mathrm{D}}{ }^{25}=-84.5\left(\mathrm{c} 1.1, \mathrm{CH}_{2} \mathrm{Cl}_{2}\right) ; \mathrm{IR}(\mathrm{KBr}, v$ $\left.\mathrm{cm}^{-1}\right): 3415,3055-2975,2387-2348,1638,1618,1450,1435,1105,1068,1024 ;{ }^{1} \mathrm{H}$ $\operatorname{NMR}\left(\mathrm{CDCl}_{3}\right): \delta(\mathrm{ppm}) 0.50-2.70(3 \mathrm{H}, \mathrm{br}), 1.28\left(3 \mathrm{H}, \mathrm{d},{ }^{3} J_{\mathrm{H}-\mathrm{H}}=6.9\right), 1.83(1 \mathrm{H}, \mathrm{d}, J=$ $4.0), 2.61\left(3 \mathrm{H}, \mathrm{d},{ }^{3} J_{\mathrm{P}-\mathrm{H}}=7.4\right), 4.47(1 \mathrm{H}, \mathrm{m}), 4.98\left(1 \mathrm{H}, \mathrm{d},{ }^{3} J_{\mathrm{H}-\mathrm{H}}=5.6\right), 7.00-7.75(14 \mathrm{H}$, $\mathrm{m}), 7.86\left(1 \mathrm{H}, \mathrm{d}, J_{\mathrm{H}-\mathrm{H}}=8.1\right), 7.95\left(1 \mathrm{H}, \mathrm{d}, J_{\mathrm{H}-\mathrm{H}}=7.9\right), 8.23\left(1 \mathrm{H}, \mathrm{d}, J_{\mathrm{H}-\mathrm{H}}=8.4\right) ;{ }^{13} \mathrm{C} \mathrm{NMR}$ $\left(\mathrm{CDCl}_{3}\right): \delta(\mathrm{ppm}) 11.6\left(\mathrm{~d},{ }^{3} J_{\mathrm{P}-\mathrm{C}}=4.3\right), 31.4\left(\mathrm{~d},{ }^{2} J_{\mathrm{P}-\mathrm{C}}=3.4\right), 58.1\left(\mathrm{~d},{ }^{2} J_{\mathrm{P}-\mathrm{C}}=10.5\right), 79.1$ $\left(\mathrm{d},{ }^{3} J_{\mathrm{P}-\mathrm{C}}=2.0\right), 124.6\left(\mathrm{~d}, J_{\mathrm{P}-\mathrm{C}}=10.6\right), 126.0-126.5,127.1-129.0,130.9,131.8-132.3$, $132.2\left(\mathrm{~d}, J_{\mathrm{P}-\mathrm{C}}=10.2\right), 132.7\left(\mathrm{~d}, J_{\mathrm{P}-\mathrm{C}}=7.6\right), 133.3\left(\mathrm{~d}, J_{\mathrm{P}-\mathrm{C}}=11.4\right), 134.0\left(\mathrm{~d}, J_{\mathrm{P}-\mathrm{C}}=7.9\right)$, $142.5 ;{ }^{31} \mathrm{P} \mathrm{NMR}\left(\mathrm{CDCl}_{3}\right): \delta(\mathrm{ppm})+72.1(\mathrm{br})$. 
S5

$(R p)-(-)-N-M e t h y l[(1 R, 2 S)(2-h y d r o x y-1-p h e n y l)$ ethyl] amino-2-naphtylphenyl

phosphine borane 11e. ${ }^{20 \mathrm{a}, 31}$ Yield $=89 \%$; White crystals $(i \mathrm{PrOH} / \mathrm{Hexane}) ; \mathrm{R}_{\mathrm{f}}=0.55$ (toluene/AcOEt 9:1); $\mathrm{mp}=136^{\circ} \mathrm{C} ;[\alpha]_{\mathrm{D}}{ }^{25}=-64.7\left(\mathrm{c} 1.1, \mathrm{CH}_{2} \mathrm{Cl}_{2}\right)$. IR $\left(\mathrm{KBr}, v \mathrm{~cm}^{-1}\right)$ : $3523,3052-2955,2374,1455,1436,1108,1062,1022 ;{ }^{1} \mathrm{H} \mathrm{NMR}\left(\mathrm{CDCl}_{3}\right): \delta(\mathrm{ppm}) 0.00-$ $2.00(3 \mathrm{H}$, br $), 1.27\left(3 \mathrm{H}, \mathrm{d},{ }^{3} J_{\mathrm{H}-\mathrm{H}}=6.9\right), 1.85(1 \mathrm{H}, \mathrm{br}), 2.52\left(3 \mathrm{H}, \mathrm{d},{ }^{3} J_{\mathrm{P}-\mathrm{H}}=7.8\right), 4.35$ $(1 \mathrm{H}, \mathrm{m}), 4.84\left(1 \mathrm{H}, \mathrm{d},{ }^{3} J_{\mathrm{H}-\mathrm{H}}=5.6\right), 7.10-7.65(13 \mathrm{H}, \mathrm{m}), 7.80-7.90(3 \mathrm{H}, \mathrm{m}), 8.10(1 \mathrm{H}, \mathrm{d}$, $\left.J_{\mathrm{H}-\mathrm{H}}=12\right) ;{ }^{13} \mathrm{C} \mathrm{NMR}\left(\mathrm{CDCl}_{3}\right): \delta(\mathrm{ppm}) 13.5,30.5\left(\mathrm{~d},{ }^{2} J_{\mathrm{P}-\mathrm{C}}=3.8\right), 58.2\left(\mathrm{~d},{ }^{2} J_{\mathrm{P}-\mathrm{C}}=10.3\right)$, $78.7\left(\mathrm{~d},{ }^{3} J_{\mathrm{P}-\mathrm{C}}=5.7\right), 126.7,127.6-129.0,130.6\left(\mathrm{~d}, J_{\mathrm{P}-\mathrm{C}}=68.3\right), 130.7\left(\mathrm{~d}, J_{\mathrm{P}-\mathrm{C}}=2.3\right)$, $132.0\left(\mathrm{~d}, J_{\mathrm{P}-\mathrm{C}}=10.3\right), 132.6\left(\mathrm{~d}, J_{\mathrm{P}-\mathrm{C}}=11.5\right), 133.8\left(\mathrm{~d}, J_{\mathrm{P}-\mathrm{C}}=10.7\right), 134.3,142.4 ; 31 \mathrm{P}$ $\operatorname{NMR}\left(\mathrm{CDCl}_{3}\right): \delta(\mathrm{ppm})+71.7(\mathrm{br}) ; \operatorname{HRMS}(\mathrm{EI}, 70 \mathrm{eV})$ : calcd for $\mathrm{C}_{26} \mathrm{H}_{29} \mathrm{BNOP}\left[\mathrm{M}^{+}\right]$: 413.2080; Found: 413.2076; Anal. calcd for $\mathrm{C}_{26} \mathrm{H}_{29} \mathrm{BNOP}$ (413.2994): C 75.56, H 7.07, N 3.39; Found: C 75.77, H 7.09, N 3.29.

\section{$(R p)-(-)-N-m e t h y l[(1 R, 2 S)(2-h y d r o x y-1-p h e n y l)$ ethyl $]$ amino-o-biphenylphenyl}

phosphine borane 11f. ${ }^{31 \mathrm{a}}$ Yield $=81 \%$; White crystals $(\mathrm{iPrOH} / \mathrm{Hexane}) ; \quad \mathrm{R}_{\mathrm{f}}=0.30$ (toluene); $\mathrm{mp}=101^{\circ} \mathrm{C} ;[\alpha]_{\mathrm{D}}{ }^{20}=-43.4\left(\mathrm{c} 1.2, \mathrm{CHCl}_{3}\right) ; \mathrm{IR}(\mathrm{KBr}, \mathrm{v} \mathrm{cm}-1): 3547,3058-$ $2880,2422-2327,1451,1435,1163,1101,1008,914,868,773,757,707 ;{ }^{1} \mathrm{H}$ NMR $\left(\mathrm{CDCl}_{3}\right): \delta(\mathrm{ppm}) 0.10-1.60(3 \mathrm{H}, \mathrm{br}), 0.70\left(3 \mathrm{H}, \mathrm{d},{ }^{3} J_{\mathrm{H}-\mathrm{H}}=6.9\right), 1.44(1 \mathrm{H}, \mathrm{br}), 2.48(3 \mathrm{H}$, $\left.\mathrm{d},{ }^{3} J_{\mathrm{P}-\mathrm{H}}=7.2\right), 3.90(1 \mathrm{H}, \mathrm{m}), 4.78\left(1 \mathrm{H}, \mathrm{d},{ }^{3} J_{\mathrm{H}-\mathrm{H}}=2.8\right), 7.00-7.80(17 \mathrm{H}, \mathrm{m}), 7.45-7.65$ $(2 \mathrm{H}, \mathrm{m}) ;{ }^{13} \mathrm{C} \mathrm{NMR}\left(\mathrm{CDCl}_{3}\right): \delta(\mathrm{ppm}) 9.9\left(\mathrm{~d},{ }^{3} J_{\mathrm{P}-\mathrm{C}}=6.6\right), 31.7\left(\mathrm{~d},{ }^{2} J_{\mathrm{P}-\mathrm{C}}=4.1\right), 58.0(\mathrm{~d}$, $\left.{ }^{2} J_{\mathrm{P}-\mathrm{C}}=10.3\right), 78.8,125.6,126.8\left(\mathrm{~d}, J_{\mathrm{P}-\mathrm{C}}=9.8\right), 127.2\left(\mathrm{~d}, J_{\mathrm{P}-\mathrm{C}}=11.5\right), 127.4,128.1$, $128.1\left(\mathrm{~d}, J_{\mathrm{P}-\mathrm{C}}=10.1\right), 128.9\left(\mathrm{~d}, 1 J_{\mathrm{P}-\mathrm{C}}=66.5\right), 129.7,130.4-130.6,132.2\left(\mathrm{~d}, J_{\mathrm{P}-\mathrm{C}}=9.8\right)$, $132.7\left(\mathrm{~d}, J_{\mathrm{P}-\mathrm{C}}=8.4\right), 133.4\left(\mathrm{~d},{ }^{1} J_{\mathrm{P}-\mathrm{C}}=57.9\right), 134.1\left(\mathrm{~d}, J_{\mathrm{P}-\mathrm{C}}=10.0\right), 141.4\left(\mathrm{~d}, J_{\mathrm{P}-\mathrm{C}}=\right.$

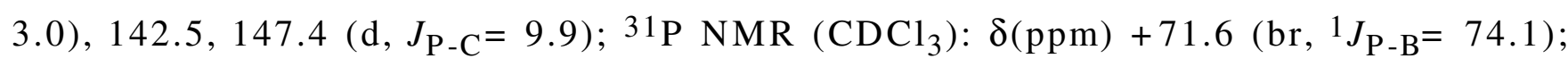
MS $\left(\mathrm{DCI}, \mathrm{CH}_{4}\right) \mathrm{m} / \mathrm{z}$ (relative intensity): $440\left(\mathrm{M}^{+}+\mathrm{H} ; 10\right), 438\left(\mathrm{M}^{+}-\mathrm{H} ; 25\right), 426\left(\mathrm{M}^{+}+\mathrm{H}-\right.$ 
$\left.\mathrm{BH}_{3} ; 25\right), 410\left(\mathrm{M}^{+}-\mathrm{H}-\mathrm{CH}_{3} ; 5\right), 318$ (10), 307 (5), 279 (40), 263 (20), 176 (20), 160 (40), 148 (100), 133 (15); Anal. calcd for $\mathrm{C}_{28} \mathrm{H}_{31} \mathrm{BNOP}$ (439.3366): C, 76.55; H, 7.11; N, 3.19; Found: C, 76.36; H, 7.22; N, 3.01 .

\section{( $S$ p) - ( -)-N-Methyl[ ( $1 R, 2 S)$ (2-hydroxy-1-phenyl) ethyl] aminocyclohexylphenyl}

phosphine borane 11g. Yield $=87 \%$; White crystals (iPrOH/Hexane); $\mathrm{R}_{\mathrm{f}}=0.26$ (toluene); $\mathrm{mp}=98^{\circ} \mathrm{C} ;[\alpha]_{\mathrm{D}}{ }^{25}=-28.5\left(\mathrm{c} 0.7, \mathrm{CHCl}_{3}\right) ; \mathrm{IR}\left(\mathrm{KBr}, \mathrm{v} \mathrm{cm}^{-1}\right): 3538,3029-2857$, $2369,1492,1452,1436,1368,1257,1221,1159,1109,1086,1000,961,887,758,742$, 695; ${ }^{1} \mathrm{H} \mathrm{NMR}\left(\mathrm{CDCl}_{3}\right): \delta(\mathrm{ppm})$ 0.10-1.60 (3H, br), $1.15\left(3 \mathrm{H}, \mathrm{d},{ }^{3} J_{\mathrm{H}-\mathrm{H}}=6.9\right), 1.20-1.90$ $(10 \mathrm{H}, \mathrm{m}), 2.27-2.34(1 \mathrm{H}, \mathrm{m}), 2.63\left(3 \mathrm{H}, \mathrm{d},{ }^{3} \mathrm{~J}_{\mathrm{P}-\mathrm{H}}=7.2\right), 4.02-4.20(1 \mathrm{H}, \mathrm{m}), 4.80(1 \mathrm{H}, \mathrm{d}$, $\left.{ }^{3} \mathrm{~J}_{\mathrm{H}-\mathrm{H}}=4.8\right), 7.10-7.65(10 \mathrm{H}, \mathrm{m}) ;{ }^{13} \mathrm{C} \mathrm{NMR}\left(\mathrm{CDCl}_{3}\right): \delta(\mathrm{ppm}) 12.2\left(\mathrm{~d},{ }^{3} J_{\mathrm{P}-\mathrm{C}}=3.8\right), 25.9$, $26.6-27.0,29.3\left(\mathrm{~d},{ }^{2} J_{\mathrm{P}-\mathrm{C}}=3.3\right), 32.6\left(\mathrm{~d},{ }^{1} J_{\mathrm{P}-\mathrm{C}}=43.7\right), 58.3\left(\mathrm{~d},{ }^{2} J_{\mathrm{P}-\mathrm{C}}=8.1\right), 78.6\left(\mathrm{~d},{ }^{3} J_{\mathrm{P}-}\right.$ $\mathrm{C}=2.3), 126.0,127.4,128.2,128.3\left(\mathrm{~d}, J_{\mathrm{P}-\mathrm{C}}=9.4\right), 130.5\left(\mathrm{~d}, J_{\mathrm{P}-\mathrm{C}}=2.1\right), 130.8\left(\mathrm{~d},{ }^{1} J_{\mathrm{P}-}\right.$ $\mathrm{C}=55.7), 131.2\left(\mathrm{~d}, J_{\mathrm{P}-\mathrm{C}}=9.1\right), 142.5 ;{ }^{31} \mathrm{P} \mathrm{NMR}\left(\mathrm{CDCl}_{3}\right): \delta(\mathrm{ppm})+73.7\left(\mathrm{br} \cdot \mathrm{d},{ }^{1} J_{\mathrm{P}-\mathrm{B}}=\right.$ 86.0); MS (DCI, $\left.\mathrm{CH}_{4}\right) \mathrm{m} / \mathrm{z}$ (relative intensity): $368\left(\mathrm{M}^{+}-\mathrm{H} ; 100\right), 356\left(\mathrm{M}^{+}+\mathrm{H}-\mathrm{BH}_{3} ; 25\right)$, 312 (10), 262 (15), 248 (15), 209 (10), 193 (25), 166 (10), 148 (20); HRMS (DCI, $\left.\mathrm{CH}_{4}\right)$ : calcd for $\mathrm{C}_{22} \mathrm{H}_{32} \mathrm{BNOP}\left[\mathrm{M}^{+}-\mathrm{H}\right]$ : 368.2315; Found: 368.2319; Anal. calcd for $\mathrm{C}_{22} \mathrm{H}_{33}$ BNOP (369.2883): C, 71.55; H, 9.01; N, 3.79; Found: C, 71.71; H, 9.13; N, 3.67 .

\section{$(S p)-(+)-N-M e t h y l[(1 R, 2 S)(2-h y d r o x y-1-p h e n y l)$ ethyl $]$ amino-tert-butylphenyl}

phosphine borane 11 h. ${ }^{32}$ Yield $=82 \%$; White crystals $(i \mathrm{PrOH} /$ Hexane $) ; \mathrm{R}_{\mathrm{f}}=0.10$ (toluene); $\mathrm{mp}=92{ }^{\circ} \mathrm{C} ;[\alpha]_{\mathrm{D}^{25}}=+3.8\left(\mathrm{c} 1.0, \mathrm{CHCl}_{3}\right) ; \mathrm{IR}\left(\mathrm{KBr}, v \mathrm{~cm}^{-1}\right): 3581,3050-$ $2984,2380,1449,1433,1384,1180,1102,1071,1028,981,948,763,742,719,699 ;{ }^{1} \mathrm{H}$ $\operatorname{NMR}\left(\mathrm{CDCl}_{3}\right): \delta(\mathrm{ppm}) 0.00-1.60(3 \mathrm{H}, \mathrm{br}), 1.08\left(3 \mathrm{H}, \mathrm{d},{ }^{3} J_{\mathrm{H}-\mathrm{H}}=7.0\right), 1.22\left(9 \mathrm{H}, \mathrm{d},{ }^{3} J_{\mathrm{P}-}\right.$ $\mathrm{H}=13.8), 1.82(1 \mathrm{H}, \mathrm{br}), 2.78\left(3 \mathrm{H}, \mathrm{d},{ }^{3} J_{\mathrm{P}-\mathrm{H}}=6.4\right), 4.05(1 \mathrm{H}, \mathrm{m}), 5.09\left(1 \mathrm{H}, \mathrm{d},{ }^{3} J_{\mathrm{H}-\mathrm{H}}=\right.$ 
3.4), 7.00-7.40 (8H, m), 7.45-7.65 $(2 \mathrm{H}, \mathrm{m}) ;{ }^{13} \mathrm{C} \mathrm{NMR}\left(\mathrm{CDCl}_{3}\right): \delta(\mathrm{ppm}) 10.6\left(\mathrm{~d},{ }^{3} J_{\mathrm{P}-\mathrm{C}}=\right.$ $4.0), 27.8\left(\mathrm{~d},{ }^{2} J_{\mathrm{P}-\mathrm{C}}=2.6\right), 33.6\left(\mathrm{~d},{ }^{2} J_{\mathrm{P}-\mathrm{C}}=4.6\right), 34.6\left(\mathrm{~d},{ }^{1} J_{\mathrm{P}-\mathrm{C}}=34.6\right), 58.5\left(\mathrm{~d},{ }^{2} J_{\mathrm{P}-\mathrm{C}}=\right.$ $9.1), 79.8\left(\mathrm{~d},{ }^{3} J_{\mathrm{P}-\mathrm{C}}=1.8\right), 125.8,127.3,128.2,128.4\left(\mathrm{~d}, J_{\mathrm{P}-\mathrm{C}}=9.6\right), 130.1\left(\mathrm{~d}, J_{\mathrm{P}-\mathrm{C}}=\right.$ $\left.2.1), 131.6\left(\mathrm{~d}, J_{\mathrm{P}-\mathrm{C}}=8.7\right), 132.5\left(\mathrm{~d},{ }^{1} J_{\mathrm{P}-\mathrm{C}}=55.8\right), 142.8 ;{ }^{31} \mathrm{P} \mathrm{NMR}_{(\mathrm{CDCl}}\right): \delta(\mathrm{ppm})$ $+87.5\left(\right.$ br.d,$\left.{ }^{1} J_{\mathrm{P}-\mathrm{B}}=84.9\right) ; \mathrm{MS}\left(\mathrm{DCI}, \mathrm{CH}_{4}\right): \mathrm{m} / \mathrm{z} 342\left(\mathrm{M}^{+}-\mathrm{H} ; 90\right), 330\left(\mathrm{M}^{+}+\mathrm{H}-\mathrm{BH}_{3} ; 50\right)$, 312 (10), 272 (15), 236 (25), 222 (20), 183 (50), 167 (40), 148 (100); HRMS (DCI, $\left.\mathrm{CH}_{4}\right)$ : calcd for $\mathrm{C}_{20} \mathrm{H}_{30} \mathrm{BNOP}\left[\mathrm{M}^{+}-\mathrm{H}\right]$ : 342.2158; Found: 342.2162; Anal. calcd for $\mathrm{C}_{20} \mathrm{H}_{31}$ BNOP (343.2510): C 69.98, H 9.10, N 4.08; Found: C 69.69, H 9.31, N 3.97.

Structure and crytal data forthe aminophosphine borane $11 \mathrm{~h}$

Figure $S 1$
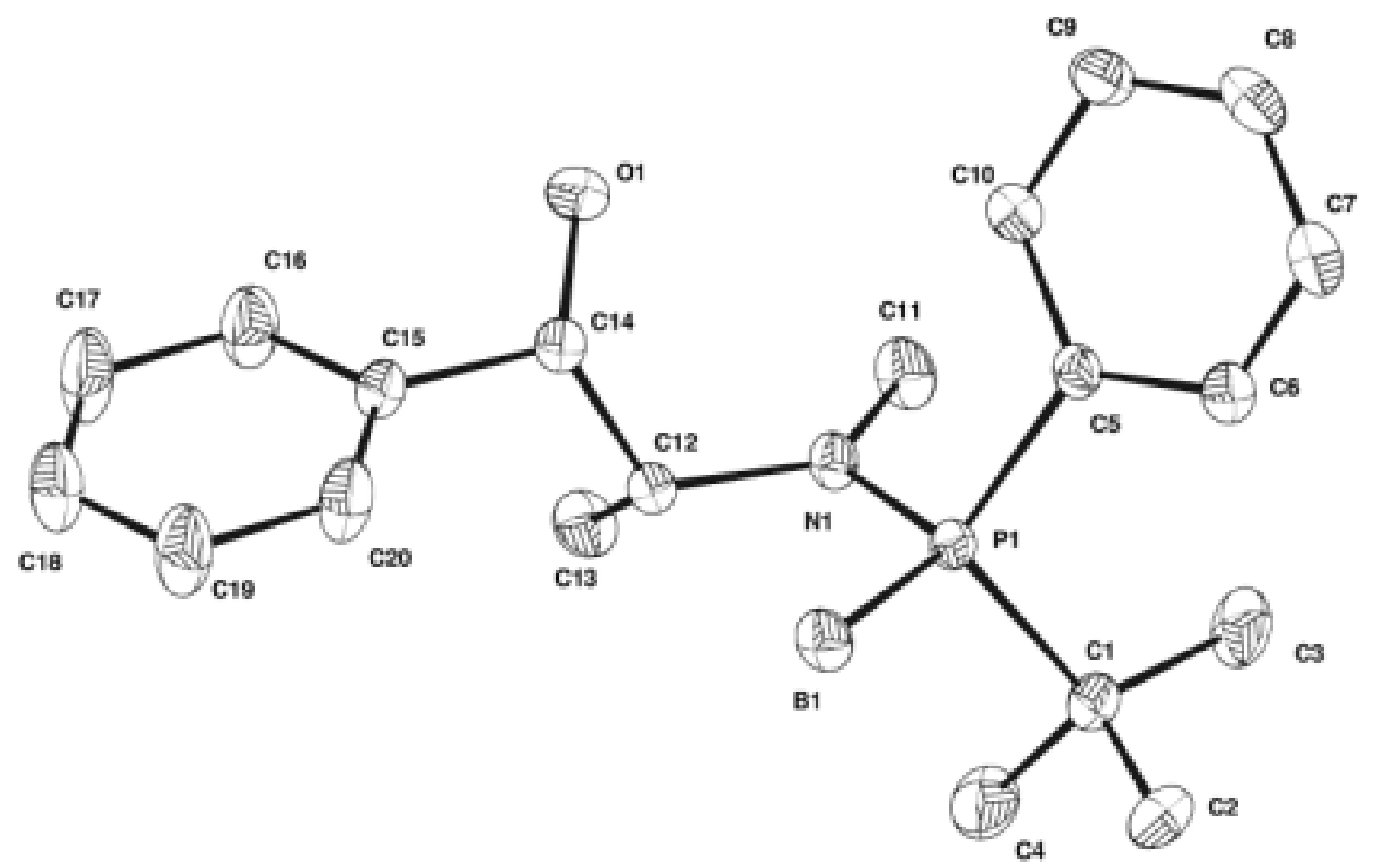

\section{TABLE S1}

\section{Formula}

Fw =

Crystal System

a $(\AA)=$
$\mathrm{C}_{20} \mathrm{H}_{31} \mathrm{~B}_{1} \mathbf{N}_{1} \mathrm{O}_{1} \mathbf{P}_{1}$

346.26

Orthorhombic

$7.194(5)$ 
b $(\stackrel{\AA}{\AA})=$

c $(\AA)=$

$\alpha$ (deg.) $=$

$\beta$ (deg.) =

$\gamma($ deg. $)=$

$\mathrm{V}\left(\AA^{3}\right)=$

$\mathrm{Z}=$

Space group

Crystal shape

Crystal colour

Linear absorption coefficient $\mu\left(\mathrm{cm}^{-1}\right)$

Density $\rho\left(\mathrm{g} \mathrm{cm}^{3}\right)$

Diffractometer

Radiation

Scan type

Scan range $\left({ }^{\circ}\right)$

$\theta$ Limits $\left({ }^{\circ}\right)$

Temperature of measurement

Octants collected

$\mathrm{Nb}$ of data collected

$\mathrm{Nb}$ of unique data used for refinement

Decay of standards reflections \%

$\mathbf{R}=\Sigma \mid$ Fo $-\mid$ Fc $\| / \Sigma$ Fo

$\mathrm{Rw}^{*}=\left[\Sigma \mathrm{w}(\text { Fo }-\mid \text { Fc } \mid)^{2} / \Sigma \mathrm{wFo}^{2}\right]^{1 / 2}$

Absorption correction

Secondary extinction coefficient

Goodness of fit

Nb of variables

$\Delta$ pmin $\left(\mathrm{e} / \AA^{3}\right)$

$\Delta \rho \max \left(\mathrm{e} / \AA^{3}\right)$
$11.967(2)$

23.749 (5)

90.

90.

90.

2044 (2)

4

P $2_{1} 2_{1} 2_{1}$

Parallelepiped

colorless

1.355

1.115

MACH 3-Enraf Nonius

$\operatorname{MoK} \alpha(\lambda=0.71069 \AA)$

$\omega / 2 \theta$

$0.8+0.345 \operatorname{tg} \theta$

1 - 30

Room temperature

0,$10 ; 0,16 ; 0,33$

3409

$2504(\mathrm{Fo})^{2}>3 \sigma(\mathrm{Fo})^{2}$

3.6

0.0495

0.0585

Psiscan $(\min =1.00, \max =1.14)$

321.6

0.942

219

$-0.21$

0.39

* Weighting scheme of the form $w=w^{\prime}\left[1-((|| F o|-| F c||) / 6 \sigma(F o))^{2}\right]^{2}$ with $w^{\prime}=1 / \Sigma_{\mathbf{r}} A_{\mathbf{r}} T_{\mathbf{r}}(X)$ with coefficients 10.7,1.23 and 8.22 for a Chebyshev serie for which $\mathrm{X}=\mathrm{Fo} / \mathrm{Fo}$ (max)

Table S2 : Anisotropic thermal parameters for $\mathrm{C}_{20} \mathrm{H}_{31} \mathrm{~B}_{1} \mathrm{~N}_{1} \mathrm{O}_{1} \mathrm{P}_{1}$

$\begin{array}{llllllr}\text { Atom } & \mathrm{U}(11) & \mathrm{U}(22) & \mathrm{U}(33) & \mathrm{U}(23) & \mathrm{U}(13) & \mathrm{U}(12) \\ \mathrm{P}(1) & 0.0438(3) & 0.0334(2) & 0.0406(3) & -0.0002(2) & -0.0000(2) & 0.0054(2) \\ \mathrm{N}(1) & 0.045(1) & 0.0368(9) & 0.051(1) & 0.0004(8) & -0.0093(9) & 0.0066(9) \\ \mathrm{O}(1) & 0.096(2) & 0.061(1) & 0.049(1) & 0.0091(9) & 0.003(1) & 0.002(1) \\ \mathrm{C}(1) & 0.073(2) & 0.046(1) & 0.057(1) & -0.010(1) & 0.016(1) & 0.001(1) \\ \mathrm{C}(2) & 0.133(4) & 0.076(2) & 0.052(2) & -0.017(1) & 0.007(2) & 0.001(3) \\ \mathrm{C}(3) & 0.081(2) & 0.059(2) & 0.102(3) & -0.020(2) & 0.014(2) & 0.021(2) \\ \mathrm{C}(4) & 0.106(3) & 0.089(3) & 0.103(3) & -0.028(2) & 0.058(3) & -0.026(3) \\ \mathrm{C}(5) & 0.046(1) & 0.036(1) & 0.050(1) & 0.0010(9) & 0.002(1) & 0.005(1) \\ \mathrm{C}(6) & 0.055(1) & 0.048(1) & 0.059(2) & -0.001(1) & -0.004(1) & 0.001(1) \\ \mathrm{C}(7) & 0.054(2) & 0.045(1) & 0.093(2) & 0.000(1) & -0.003(2) & -0.003(1)\end{array}$




$\begin{array}{lllllrr}\text { C (8) } & 0.063(2) & 0.052(1) & 0.093(2) & 0.022(2) & 0.015(2) & -0.000(1) \\ \text { C (9) } & 0.079(2) & 0.059(2) & 0.063(2) & 0.011(1) & 0.014(2) & -0.000(2) \\ \text { C (10) } & 0.065(2) & 0.047(1) & 0.055(1) & 0.004(1) & 0.001(1) & -0.002(1) \\ \text { C (11) } & 0.057(2) & 0.051(1) & 0.080(2) & 0.008(1) & -0.017(2) & 0.014(1) \\ \text { C (12) } & 0.045(1) & 0.039(1) & 0.044(1) & 0.0003(9) & 0.0008(9) & -0.002(1) \\ \text { C(13) } & 0.045(1) & 0.065(2) & 0.082(2) & 0.002(2) & 0.007(1) & -0.010(1) \\ \text { C(14) } & 0.053(1) & 0.043(1) & 0.049(1) & -0.0024(9) & 0.003(1) & -0.001(1) \\ \text { C(15) } & 0.056(1) & 0.044(1) & 0.053(1) & -0.009(1) & 0.001(1) & 0.004(1) \\ \text { C(16) } & 0.104(3) & 0.058(2) & 0.089(3) & -0.010(2) & -0.040(2) & -0.004(2) \\ \text { C(17) } & 0.129(4) & 0.055(2) & 0.120(4) & -0.023(2) & -0.049(3) & -0.007(2) \\ \text { C(18) } & 0.100(3) & 0.045(1) & 0.121(3) & -0.014(2) & -0.015(3) & 0.010(2) \\ \text { C(19) } & 0.124(4) & 0.050(2) & 0.113(3) & -0.011(2) & -0.045(3) & 0.026(2) \\ \text { C (20) } & 0.098(3) & 0.051(2) & 0.091(2) & -0.018(2) & -0.038(2) & 0.016(2) \\ \text { B(1) } & 0.054(2) & 0.047(1) & 0.062(2) & 0.007(1) & -0.014(1) & 0.009(1)\end{array}$

Table s3 : Hydrogen atoms fractional atomic coordinates for

\begin{tabular}{lcccc} 
& \multicolumn{4}{c}{$\mathrm{C}_{2} \mathrm{H}_{31} \mathrm{~B}_{1} \mathrm{~N}_{1} \mathrm{O}_{1} \mathrm{P}_{1}$} \\
Atom & $\mathrm{x} / \mathrm{a}$ & $\mathrm{y} / \mathrm{b}$ & $\mathrm{z} / \mathrm{C}$ & $\mathrm{U}(\mathrm{iso})$ \\
$\mathrm{H}(21)$ & 0.2813 & 0.7972 & 0.6869 & $0.098(3)$ \\
$\mathrm{H}(22)$ & 0.0994 & 0.7962 & 0.7280 & $0.098(3)$ \\
$\mathrm{H}(23)$ & 0.1818 & 0.9146 & 0.7047 & $0.098(3)$ \\
$\mathrm{H}(31)$ & 0.4735 & 0.6928 & 0.7521 & $0.098(3)$ \\
$\mathrm{H}(32)$ & 0.3008 & 0.6804 & 0.7963 & $0.098(3)$ \\
$\mathrm{H}(33)$ & 0.4983 & 0.7330 & 0.8174 & $0.098(3)$ \\
$\mathrm{H}(41)$ & 0.5862 & 0.8816 & 0.7245 & $0.098(3)$ \\
$\mathrm{H}(42)$ & 0.4723 & 0.9937 & 0.7431 & $0.098(3)$ \\
$\mathrm{H}(43)$ & 0.5929 & 0.9244 & 0.7898 & $0.098(3)$ \\
$\mathrm{H}(61)$ & -0.0182 & 0.7282 & 0.7969 & $0.098(3)$ \\
$\mathrm{H}(71)$ & -0.2133 & 0.6067 & 0.8513 & $0.098(3)$ \\
$\mathrm{H}(81)$ & -0.2330 & 0.6257 & 0.9492 & $0.098(3)$ \\
$\mathrm{H}(91)$ & -0.0678 & 0.7718 & 0.9955 & $0.098(3)$ \\
$\mathrm{H}(101)$ & 0.1280 & 0.8944 & 0.9420 & $0.098(3)$ \\
$\mathrm{H}(111)$ & 0.5752 & 0.9312 & 0.9302 & $0.098(3)$ \\
$\mathrm{H}(112)$ & 0.4179 & 0.8333 & 0.9251 & $0.098(3)$ \\
$\mathrm{H}(113)$ & 0.5738 & 0.8480 & 0.8757 & $0.098(3)$ \\
$\mathrm{H}(121)$ & 0.3571 & 1.1236 & 0.8465 & $0.098(3)$ \\
$\mathrm{H}(131)$ & 0.6251 & 1.1968 & 0.8927 & $0.098(3)$ \\
$\mathrm{H}(132)$ & 0.6758 & 1.0905 & 0.8524 & $0.098(3)$ \\
$\mathrm{H}(133)$ & 0.6621 & 1.0740 & 0.9205 & $0.098(3)$ \\
$\mathrm{H}(141)$ & 0.1550 & 1.1034 & 0.9284 & $0.098(3)$ \\
$\mathrm{H}(161)$ & 0.4504 & 1.2835 & 0.9998 & $0.098(3)$ \\
$\mathrm{H}(171)$ & 0.4539 & 1.4851 & 0.9937 & $0.098(3)$ \\
$\mathrm{H}(181)$ & 0.2704 & 1.5733 & 0.9268 & $0.098(3)$ \\
$\mathrm{H}(191)$ & 0.1088 & 1.4717 & 0.8571 & $0.098(3)$ \\
$\mathrm{H}(201)$ & 0.1062 & 1.2700 & 0.8637 & $0.098(3)$
\end{tabular}


S10

Table S4 : Fractional atomic coordinates for $\mathrm{C}_{20} \mathrm{H}_{31} \mathrm{~B}_{1} \mathrm{~N}_{1} \mathrm{O}_{1} \mathrm{P}_{1}$

\begin{tabular}{|c|c|c|c|c|}
\hline Atom & $\mathrm{x} / \mathrm{a}$ & $y / b$ & $z / c$ & $\mathrm{U}$ ( eqv) \\
\hline$P(1)$ & $0.21862(9)$ & $0.92082(5)$ & $0.82643(2)$ & 0.0387 \\
\hline $\mathrm{N}(1)$ & $0.3721(3)$ & $0.9660(2)$ & $0.87380(9)$ & 0.0429 \\
\hline$O(1)$ & $0.3445(4)$ & $1.0853(2)$ & $0.98352(9)$ & 0.0654 \\
\hline$C(1)$ & $0.3426(5)$ & $0.8452(2)$ & $0.7691(1)$ & 0.0557 \\
\hline$C(2)$ & $0.2164(9)$ & $0.8372(4)$ & $0.7171(1)$ & 0.0784 \\
\hline$C(3)$ & $0.4076(6)$ & $0.7269(3)$ & $0.7851(2)$ & 0.0726 \\
\hline$C(4)$ & $0.5126(8)$ & $0.9173(4)$ & $0.7547(2)$ & 0.0844 \\
\hline$C(5)$ & $0.0743(4)$ & $0.8215(2)$ & $0.8652(1)$ & 0.0436 \\
\hline$C(6)$ & $-0.0282(4)$ & $0.7375(2)$ & $0.8389(1)$ & 0.0536 \\
\hline$C(7)$ & $-0.1402(5)$ & $0.6669(2)$ & $0.8697(2)$ & 0.0606 \\
\hline$C(8)$ & $-0.1537(5)$ & $0.6788(3)$ & $0.9270(2)$ & 0.0638 \\
\hline$C(9)$ & $-0.0576(6)$ & $0.7626(3)$ & $0.9537(1)$ & 0.0648 \\
\hline$C(10)$ & $0.0568(5)$ & $0.8337(2)$ & $0.9226(1)$ & 0.0553 \\
\hline$C(11)$ & $0.4922(5)$ & $0.8890(3)$ & $0.9040(2)$ & 0.0579 \\
\hline$C(12)$ & $0.4030(4)$ & $1.0873(2)$ & $0.8821(1)$ & 0.0424 \\
\hline$C(13)$ & $0.6090(4)$ & $1.1154(3)$ & $0.8881(2)$ & 0.0613 \\
\hline$C(14)$ & $0.2867(5)$ & $1.1320(2)$ & $0.9310(1)$ & 0.0481 \\
\hline$C(15)$ & $0.2836(5)$ & $1.2590(2)$ & $0.9316(1)$ & 0.0497 \\
\hline$C(16)$ & $0.3796(7)$ & $1.3221(3)$ & $0.9694(2)$ & 0.0757 \\
\hline$C(17)$ & $0.3764(9)$ & $1.4386(3)$ & $0.9669(2)$ & 0.0854 \\
\hline$C(18)$ & $0.2756(8)$ & $1.4915(3)$ & $0.9261(2)$ & 0.0795 \\
\hline$C(19)$ & $0.1790(8)$ & $1.4308(3)$ & $0.8882(2)$ & 0.0812 \\
\hline$C(20)$ & $0.1797(6)$ & $1.3147(3)$ & $0.8912(2)$ & 0.0703 \\
\hline $\mathrm{B}(1)$ & $0.0676(5)$ & $1.0399(3)$ & $0.7965(1)$ & 0.0521 \\
\hline
\end{tabular}

Table S5 : Interatomic distances ( ) for $\mathrm{C}_{20} \mathrm{H}_{31} \mathrm{~B}_{1} \mathrm{~N}_{1} \mathrm{O}_{1} \mathrm{P}_{1}$

$\begin{array}{llllll}P(1) & -\mathrm{N}(1) & 1.666(2) & \mathrm{P}(1) & -\mathrm{C}(1) & 1.863(3) \\ \mathrm{P}(1) & -\mathrm{C}(5) & 1.827(3) & \mathrm{P}(1)-\mathrm{B}(1) & 1.927(3) \\ \mathrm{N}(1) & -\mathrm{C}(11) & 1.452(3) & \mathrm{N}(1)-\mathrm{C}(12) & 1.481(3) \\ \mathrm{O}(1) & -\mathrm{C}(14) & 1.428(3) & \mathrm{C}(1)-\mathrm{C}(2) & 1.535(6) \\ \mathrm{C}(1)-\mathrm{C}(3) & 1.539(5) & \mathrm{C}(1)-\mathrm{C}(4) & 1.535(6) \\ \mathrm{C}(5)-\mathrm{C}(6) & 1.395(4) & \mathrm{C}(5)-\mathrm{C}(10) & 1.378(4) \\ \mathrm{C}(6) & -\mathrm{C}(7) & 1.378(5) & \mathrm{C}(7)-\mathrm{C}(8) & 1.371(5) \\ \mathrm{C}(8) & -\mathrm{C}(9) & 1.373(5) & \mathrm{C}(9)-\mathrm{C}(10) & 1.395(4) \\ \mathrm{C}(12)-\mathrm{C}(13) & 1.526(4) & \mathrm{C}(12)-\mathrm{C}(14) & 1.529(4) \\ \mathrm{C}(14)-\mathrm{C}(15) & 1.520(4) & \mathrm{C}(15)-\mathrm{C}(16) & 1.361(4) \\ \mathrm{C}(15)-\mathrm{C}(20) & 1.387(5) & \mathrm{C}(16)-\mathrm{C}(17) & 1.396(5) \\ \mathrm{C}(17)-\mathrm{C}(18) & 1.365(6) & \mathrm{C}(18)-\mathrm{C}(19) & 1.349(6) \\ \mathrm{C}(19)-\mathrm{C}(20) & 1.391(5) & & & \end{array}$

Table $\mathrm{S6}$ : Bond angles (i) for $\mathrm{C}_{20} \mathrm{H}_{31} \mathrm{~B}_{1} \mathrm{~N}_{1} \mathrm{O}_{1} \mathrm{P}_{1}$

$\begin{array}{llllll}\mathrm{N}(1) & -\mathrm{P}(1) & -\mathrm{C}(1) & 109.5(1) & \mathrm{N}(1)-\mathrm{P}(1)-\mathrm{C}(5) \\ \mathrm{C}(1) & -\mathrm{P}(1) & -\mathrm{C}(5) & 108.9(1) & \mathrm{N}(1)-\mathrm{P}(1)-\mathrm{B}(1)\end{array}$




$C(1)-P(1)-B(1)$
$P(1)-C(1)-C(11)$
$C(11)-C(1)-C(12)$
$P(1)-C(1)-C(3)$
$P(1)-C(1)-C(4)$
$C(3)-C(1)-C(4)$
$P(1)-C(5)-C(10)$
$C(5)-C(6)-C(7)$
$C(7)-C(8)-C(9)$
$C(5)-C(10)-C(9)$
$N(1)-C(12)-C(14)$
$O(1)-C(14)-C(12)$
$C(12)-C(14)-C(15)$
$C(14)-C(15)-C(20)$
$C(15)-C(16)-C(17)$
$C(17)-C(18)-C(19)$
$C(15)-C(20)-C(19)$

$111.1(2)$

$121.5(2)$

$117.8(2)$

$114.3(2)$

$105.7(2)$

$109.3(4)$

$118.8(2)$

$120.9(3)$

$119.9(3)$

$120.9(3)$

$111.2(2)$

$111.5(2)$

$111.4(2)$

$118.8(3)$

$121.2(4)$

$119.8(3)$

$121.1(3)$

S11

$$
\begin{aligned}
& \mathrm{C}(5)-\mathrm{P}(1)-\mathrm{B}(1) \\
& \mathrm{P}(1)-\mathrm{N}(1)-\mathrm{C}(12)
\end{aligned}
$$

$P(1)-C(1)-C(2)$

$C(2)-C(1)-C(3)$

$C(2)-C(1)-C(4)$

$P(1)-C(5)-C(6)$

$C(6)-C(5)-C(10)$

$C(6)-C(7)-C(8)$

$C(8)-C(9)-C(10)$

$\mathrm{N}(1)-\mathrm{C}(12)-\mathrm{C}(13)$

$C(13)-C(12)-C(14)$

$O(1)-C(14)-C(15)$

$C(14)-C(15)-C(16)$

$C(16)-C(15)-C(20)$

$C(16)-C(17)-C(18)$

$C(18)-C(19)-C(20)$
$110.3(1)$

$120.5(2)$

$109.5(3)$

108.7 (3)

$109.1(3)$

$123.0(2)$

118.1 (3)

$120.4(3)$

$119.9(3)$

$112.0(2)$

$112.6(2)$

$112.8(2)$

$123.6(3)$

$117.6(3)$

120.1 (4)

$120.1(4)$

Stucture and crystal data for the $(R)$-o-anisylphenyl-o-tolylphosphine 14 .

Figure S2

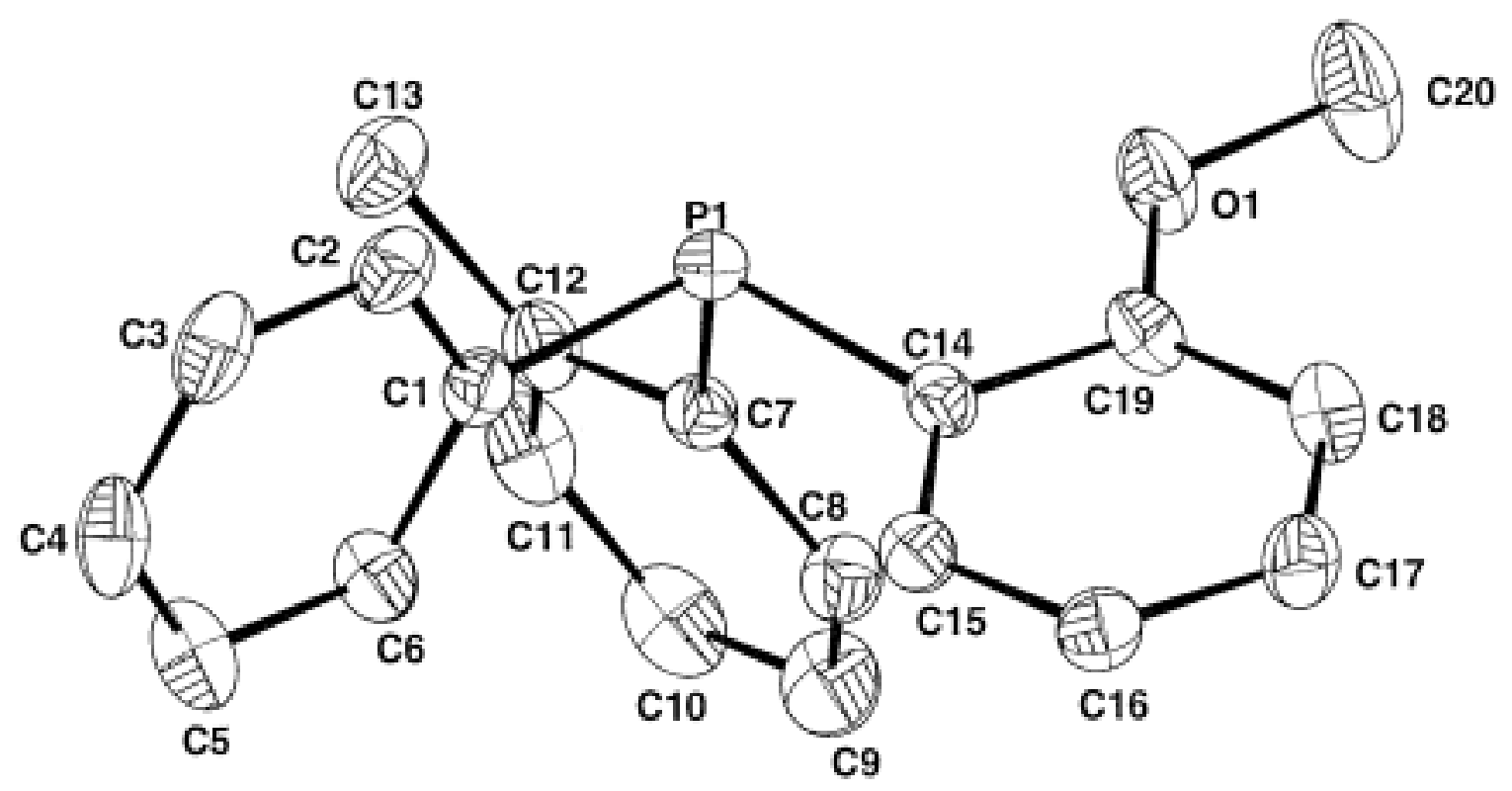


Table S7

CRYSTAL DATA for

Fw =

a $(\AA \AA)=$

b $(\dot{A})=$

c $(\hat{\mathbf{A}})=$

$\alpha\left({ }^{\circ}\right)=$

$\beta\left({ }^{\circ}\right)=$

$\gamma\left({ }^{\circ}\right)=$

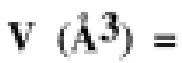

$\mathrm{Z}=$

Crystal system

Space group

Crystal shape

Crystal colour

Crystal size

Linear absorption coefficient $\mu\left(\mathrm{cm}^{-1}\right)$

Density $\rho\left(\mathrm{g} \mathrm{cm}^{-3}\right)$

Diffractometer

Radiation

Scan type

Scan range $\left({ }^{\circ}\right)$

$\theta$ Limits ( ${ }^{\circ}$ )

Temperature of measurement

Octants collected

$\mathrm{Nb}$ of data collected

$\mathrm{Nb}$ of unique data collected

$\mathrm{Nb}$ of unique data used for refinement

$R$ (int)

Decay of standards reflexions \%

$\mathbf{R}=\Sigma\left\|\mathrm{Fo}_{0}|-| \mathrm{Fc}\right\| / \Sigma\left|\mathrm{Fo}_{\mathrm{O}}\right|$

$\mathbf{R w} *=\left[\Sigma \mathbf{w}(|\mathbf{F o}|-|\mathbf{F c}|)^{2 / \Sigma} \mathbf{w F o} o^{2}\right]^{1 / 2}$

Absorption correction

Extinction parameter

Goodness of fit

$\Delta \rho \min \left(\mathrm{e} / \AA^{3}\right)$

$\Delta \rho \max \left(\mathrm{e} / \AA^{3}\right)$

\section{$\mathrm{C}_{20} \mathrm{H}_{19} \mathrm{OP}$}

306.34

$7.809(1)$

7.910(1)

$13.990(2)$

90

94.30 (1)

90.

$861.8(2)$

2

Monoclinic

P 21

Parallelepiped

Colourless

$0.2,0.2,0.4$

\section{2}

1.53

CAD4 - Enraf-Nonius

$\operatorname{MoK} \alpha(\lambda=0.71069 \AA)$

$\omega / 2 \theta$

$0.8+0.345 \operatorname{tg} \theta$

$1-28$

Room temperature

0,$10 ; 0,10 ;-18,18$

2396

2234

$1198(\mathrm{Fo})^{2}>3 \sigma(\mathrm{Fo})^{2}$

.020

4

0.046

0.054

Psiscan

519

1.0

$-0.17$

0.17

* Weighting scheme of the form $w=w^{\prime}\left[1-(\text { (II Fo | - | Fe || ) / } 6 \sigma(\text { Fo }))^{2}\right]^{2}$ with $w^{\prime}=1 / \Sigma_{r} A_{r} T_{r}(X)$ with coefficients $8.12,-4.17$ and 5.45 for a Chebyshev serie for which $\mathrm{X}=\mathrm{Fo} / \mathrm{Fo}$ (max)

Table S8: Fractional atomic coordinates for: (compound 14)

$\begin{array}{lllll}\text { Atom } & \mathrm{X} / \mathrm{a} & \mathrm{y} / \mathrm{b} & \mathrm{z} / \mathrm{c} & \mathrm{U}(\mathrm{eqv}) \\ \mathrm{P}(1) & 0.2607(2) & 0.2911(4) & 0.1975(1) & 0.0511 \\ \mathrm{O}(1) & 0.1865(7) & 0.0329(9) & 0.0598(4) & 0.0723\end{array}$

$\mathrm{O}(1)$

$0.1865(7) \quad 0.0329(9) \quad 0.0598(4)$

0.0723 


$\begin{array}{lllll}\mathrm{C}(1) & 0.3969(9) & 0.425(1) & 0.2787(5) & 0.0514 \\ \mathrm{C}(2) & 0.461(1) & 0.574(1) & 0.2419(6) & 0.0643 \\ \mathrm{C}(3) & 0.558(1) & 0.683(1) & 0.3001(8) & 0.0704 \\ \mathrm{C}(4) & 0.590(1) & 0.650(1) & 0.3944(7) & 0.0694 \\ \mathrm{C}(5) & 0.531(1) & 0.505(1) & 0.4327(6) & 0.0772 \\ \mathrm{C}(6) & 0.433(1) & 0.393(1) & 0.3746(5) & 0.0612 \\ \mathrm{C}(7) & 0.1503(9) & 0.163(1) & 0.2824(5) & 0.0506 \\ \mathrm{C}(8) & 0.190(1) & -0.003(1) & 0.3020(6) & 0.0625 \\ \mathrm{C}(9) & 0.100(1) & -0.097(1) & 0.3645(7) & 0.0821 \\ \mathrm{C}(10) & -0.031(1) & -0.025(2) & 0.4111(7) & 0.0775 \\ \mathrm{C}(11) & -0.074(1) & 0.144(2) & 0.3917(6) & 0.0742 \\ \mathrm{C}(12) & 0.015(1) & 0.239(1) & 0.3286(6) & 0.0595 \\ \mathrm{C}(13) & -0.036(1) & 0.421(1) & 0.3114(7) & 0.0813 \\ \mathrm{C}(14) & 0.4178(9) & 0.142(1) & 0.1556(4) & 0.0499 \\ \mathrm{C}(15) & 0.5923(9) & 0.144(1) & 0.1826(5) & 0.0563 \\ \mathrm{C}(16) & 0.7050(9) & 0.038(1) & 0.1398(5) & 0.0622 \\ \mathrm{C}(17) & 0.647(1) & -0.072(1) & 0.0715(6) & 0.0639 \\ \mathrm{C}(18) & 0.476(1) & -0.078(1) & 0.0429(5) & 0.0678 \\ \mathrm{C}(19) & 0.358(1) & 0.029(1) & 0.0844(5) & 0.0590 \\ \mathrm{C}(20) & 0.117(1) & -0.069(2) & -0.0148(9) & 0.1047\end{array}$

Table S9: anisotropic thermal parameters for: (compound 14)

\begin{tabular}{|c|c|c|c|c|c|c|}
\hline Atcm & $v(11)$ & $\nabla(22)$ & $0(33)$ & $\tau(23)$ & $\nabla(13)$ & $\nabla(12)$ \\
\hline$P(1)$ & $0.060(1)$ & $0.051(1)$ & $0.0440(8)$ & $0.003(1)$ & $-0.0015(7)$ & $0.003(1)$ \\
\hline$o(1)$ & $0.058(3)$ & $0.10 B(5)$ & $0.075(4)$ & $-0.039\{4\}$ & $-0.005(3)$ & $-0.004(4)$ \\
\hline$c(1)$ & $0.055(4)$ & $0.047(5)$ & $0.053(4)$ & $-0.004\{4\}$ & $0.004(3)$ & $0.003(4)$ \\
\hline$c(2)$ & $0.083(6)$ & $0.047(5)$ & $0.072(5)$ & $0.008(4)$ & $0.014(4)$ & $0.001(5)$ \\
\hline$c(3)$ & $0.087(6)$ & $0.043(5)$ & $0.110(7)$ & $-0.006(5)$ & $0.037(5)$ & $-0.007(5)$ \\
\hline$c(4)$ & $0.073(6)$ & $0.071(6)$ & $0.094(7)$ & $-0.035(6)$ & $0.022(5)$ & $-0.028(5)$ \\
\hline $\begin{array}{l}c(5) \\
c(6)\end{array}$ & $\begin{array}{l}0.086(6) \\
0.078(5)\end{array}$ & $\begin{array}{l}0.101(8) \\
0.061(5)\end{array}$ & $\begin{array}{l}0.062(5) \\
0.055(4)\end{array}$ & $\begin{array}{l}-0.022(5) \\
-0.000(4)\end{array}$ & $\begin{array}{l}0.008(4) \\
0.008(4)\end{array}$ & $\begin{array}{l}-0.027(6) \\
-0.022(5)\end{array}$ \\
\hline$c(7)$ & $0.047(4)$ & $0.059(5)$ & $0.048(4)$ & -0.007 (4) & $-0.006(3)$ & -0.002 (4) \\
\hline$c(8)$ & $0.073(5)$ & $0.056(5)$ & $0.061(5)$ & 0.001 (4) & $0.006(4)$ & $-0.007(4)$ \\
\hline$C(9)$ & $0.113(8)$ & $0.065(6)$ & $0.085(7)$ & $0.010(6)$ & $0.010(6)$ & $-0.026(6)$ \\
\hline$c(10)$ & $0.090(7)$ & $0.100(9)$ & $0.070(6)$ & $0.000(6)$ & $0.008(5)$ & $-0.049(7)$ \\
\hline$c(11)$ & $0.064(5)$ & $0.114(9)$ & $0.068(5)$ & $-0.026(6)$ & $0.018(4)$ & $-0.022(6)$ \\
\hline$c(12)$ & $0.051(4)$ & $0.078(7)$ & $0.056(4)$ & $-0.015(4)$ & $0.004(4)$ & $-0.007(4)$ \\
\hline$c(13)$ & $0.074(6)$ & $0.083(8)$ & $0.097(7)$ & $-0.006(6)$ & $0.018(5)$ & $0.017(6)$ \\
\hline$c(14)$ & $0.064(5)$ & $0.055(5)$ & $0.036(3)$ & $-0.000(3)$ & $0.002(3)$ & $-0.006(4)$ \\
\hline$c(15)$ & $0.065(5)$ & $0.058(5)$ & $0.047(4)$ & $-0.001(4)$ & $-0.001(3)$ & $-0.007(4)$ \\
\hline$c(16)$ & $0.050(4)$ & $0.072(6)$ & $0.067(5)$ & $-0.004(5)$ & $0.001(4)$ & $0.004(4)$ \\
\hline$c(17)$ & $0.060(5)$ & $0.075(6)$ & $0.068(5)$ & $-0.017(5)$ & $0.016(4)$ & $0.008(5)$ \\
\hline$c(18)$ & $0.081(6)$ & $0.073(6)$ & $0.060(5)$ & $-0.023(5)$ & $0.015(4)$ & $-0.011(5)$ \\
\hline$c(19)$ & $0.061(5)$ & $0.078(6)$ & $0.045(4)$ & $-0.011(4)$ & $0.006(3)$ & $-0.005(5)$ \\
\hline$C(20)$ & $0.082(7)$ & $0.18(1)$ & $0.140(9)$ & $-0.10(1)$ & $-0.006(6)$ & $-0.023(8)$ \\
\hline
\end{tabular}

Table S10: Angles for $\mathbf{1 4}$ axis 1 atom $\mathrm{C}(1) \mathrm{C}(2)$ axis 2 atom $\mathrm{P}(1) \mathrm{C}(14)$ axis 4 atom $\mathrm{C}(12) \mathrm{C}(13)$ axis 5 atom $\mathrm{C}(7) \mathrm{P}(1)$ axis 6 atom $\mathrm{C}(20) \mathrm{O}(1)$ axis 7 atom $\mathrm{C}(19) \mathrm{C}(14)$ axis 8 atom $\mathrm{C}(1) \mathrm{P}(1)$ axis 9 atom $\mathrm{C}(14) \mathrm{C}(15)$ plane 10 atom $C(7) C(8) C(9) C(10) C(11) C(12) C(13)$ plane 11 atom $\mathrm{C}(1) \mathrm{C}(2) \mathrm{C}(3) \mathrm{C}(4) \mathrm{C}(5) \mathrm{C}(6)$ plane 12 atom C(14) C(15) C(16) C(17) C(18) C(19) Angle between axis 1 and axis 2 is 80.80 Degrees Angle between axis 4 and axis 5 is 59.94 Degrees Angle between axis 6 and axis 7 is 174.93 Degrees 
Angle between axis 8 and axis 9 is 46.61 Degrees

Angle between axis 10 and axis 11 is 77.73 Degrees

Angle between axis 10 and axis 12 is 77.49 Degrees

Angle between axis 11 and axis 12 is 88.28 Degrees

Table S11: interatomic distances $(\AA)$ and bond angles $\left({ }^{\circ}\right)$ for: (compound 14)

$\begin{array}{ll}\mathrm{P}(1)-\mathrm{C}(1) & 1.836(7) \\ \mathrm{P}(1)-\mathrm{C}(7) & 1.826(8) \\ \mathrm{P}(1)-\mathrm{C}(14) & 1.830(8) \\ \mathrm{P}(1)-\mathrm{O}(1) & 2.837(6) \\ \mathrm{O}(1)-\mathrm{C}(19) & 1.359(8) \\ \mathrm{O}(1)-\mathrm{C}(20) & 1.39(1) \\ \mathrm{C}(1)-\mathrm{C}(2) & 1.39(1) \\ \mathrm{C}(1)-\mathrm{C}(6) & 1.374(9) \\ \mathrm{C}(2)-\mathrm{C}(3) & 1.37(1) \\ \mathrm{C}(3)-\mathrm{C}(4) & 1.35(1) \\ \mathrm{C}(4)-\mathrm{C}(5) & 1.36(1) \\ \mathrm{C}(5)-\mathrm{C}(6) & 1.39(1) \\ \mathrm{C}(7)-\mathrm{C}(8) & 1.37(1) \\ \mathrm{C}(7)-\mathrm{C}(12) & 1.42(1) \\ \mathrm{C}(8)-\mathrm{C}(9) & 1.38(1) \\ \mathrm{C}(9)-\mathrm{C}(10) & 1.37(1) \\ \mathrm{C}(10)-\mathrm{C}(11) & 1.40(2) \\ \mathrm{C}(11)-\mathrm{C}(12) & 1.39(1) \\ \mathrm{C}(12)-\mathrm{C}(13) & 1.51(1) \\ \mathrm{C}(14)-\mathrm{C}(15) & 1.387(9) \\ \mathrm{C}(14)-\mathrm{C}(19) & 1.39(1) \\ \mathrm{C}(15)-\mathrm{C}(16) & 1.38(1) \\ \mathrm{C}(16)-\mathrm{C}(17) & 1.34(1) \\ \mathrm{C}(17)-\mathrm{C}(18) & 1.36(1) \\ \mathrm{C}(18)-\mathrm{C}(19) & 1.41(1) \\ \mathrm{C}(1)-\mathrm{P}(1)-C(7)\end{array}$

$\mathrm{C}(1)-\mathrm{P}(1)-\mathrm{C}(7) \quad 101.4(3)$

$\mathrm{C}(1)-\mathrm{P}(1)-\mathrm{C}(14) \quad 101.6(3)$

$\mathrm{C}(7)-\mathrm{P}(1)-\mathrm{C}(14) \quad 102.2(4)$

$\mathrm{C}(19)-\mathrm{O}(1)-\mathrm{C}(20) \quad 119.8(7)$

$\mathrm{P}(1)-\mathrm{C}(1)-\mathrm{C}(2) \quad 117.9(6)$

$\mathrm{P}(1)-\mathrm{C}(1)-\mathrm{C}(6) \quad 124.3(6)$

$\mathrm{C}(2)-\mathrm{C}(1)-\mathrm{C}(6) \quad 117.7(7)$

$\mathrm{C}(1)-\mathrm{C}(2)-\mathrm{C}(3) \quad 120.5(8)$

$\mathrm{C}(2)-\mathrm{C}(3)-\mathrm{C}(4) \quad 121.0(8)$

$\mathrm{C}(3)-\mathrm{C}(4)-\mathrm{C}(5) \quad 120.1(8)$

$\mathrm{C}(4)-\mathrm{C}(5)-\mathrm{C}(6) \quad 119.6(8)$

$\mathrm{C}(1)-\mathrm{C}(6)-\mathrm{C}(5) \quad 121.1(8)$

$\mathrm{P}(1)-\mathrm{C}(7)-\mathrm{C}(8) \quad 123.5(6)$

$\mathrm{P}(1)-\mathrm{C}(7)-\mathrm{C}(12) \quad 117.8(6)$

$\mathrm{C}(8)-\mathrm{C}(7)-\mathrm{C}(12) \quad 118.7(8)$

$\mathrm{C}(7)-\mathrm{C}(8)-\mathrm{C}(9) \quad 121.5(9)$

$\mathrm{C}(8)-\mathrm{C}(9)-\mathrm{C}(10) \quad 120.7(10)$

$\mathrm{C}(9)-\mathrm{C}(10)-\mathrm{C}(11) \quad 118.9(9)$

C(10) -C(11) -C(12) 120.9(9)

$\mathrm{C}(7)-\mathrm{C}(12)-\mathrm{C}(11) \quad 119.2(9)$

$\mathrm{C}(7)-\mathrm{C}(12)-\mathrm{C}(13) \quad 122.1(8)$

$\mathrm{C}(11)-\mathrm{C}(12)-\mathrm{C}(13) \quad 118.7(9)$

$\mathrm{P}(1)-\mathrm{C}(14)-\mathrm{C}(15) \quad 125.0(6)$

$\mathrm{P}(1)-\mathrm{C}(14)-\mathrm{C}(19) \quad 116.5(5)$

$\mathrm{C}(15)-\mathrm{C}(14)-\mathrm{C}(19) \quad 118.2(7)$

$\mathrm{C}(14)-\mathrm{C}(15)-\mathrm{C}(16) \quad 121.3(7)$

$\mathrm{C}(15)-\mathrm{C}(16)-\mathrm{C}(17) \quad 120.5(7)$

$\mathrm{C}(16)-\mathrm{C}(17)-\mathrm{C}(18) \quad 120.0(7)$

$\mathrm{C}(17)-\mathrm{C}(18)-\mathrm{C}(19) \quad 121.0(7)$

$\mathrm{O}(1)-\mathrm{C}(19)-\mathrm{C}(14) \quad 116.0(7)$

$\mathrm{O}(1)-\mathrm{C}(19)-\mathrm{C}(18) \quad 124.9(7)$

$\mathrm{C}(14)-\mathrm{C}(19)-\mathrm{C}(18) \quad 119.0(7)$

$\mathrm{P}(1)-\mathrm{C}(14)-\mathrm{C}(19)-\mathrm{O}(1) \quad 52.77$ 
$\mathrm{P}(1)-\mathrm{C}(14)-\mathrm{C}(1)-\mathrm{C}(2) \quad 80.80$

S15

$\mathrm{P}(1)-\mathrm{C}(7)-\mathrm{C}(12)-\mathrm{C}(13) \quad 59.94$

Table S12: Hydrogen atoms fractional atomic coordinates for the compound 14 :

$\begin{array}{lllll}\text { Atom } & \mathrm{x} / \mathrm{a} & \mathrm{y} / \mathrm{b} & \mathrm{z} / \mathrm{b} & \mathrm{U}(\mathrm{iso}) \\ \mathrm{H}(2) & 0.4221 & 0.5871 & 0.1682 & 0.098(7) \\ \mathrm{H}(3) & 0.5867 & 0.7833 & 0.2675 & 0.098(7) \\ \mathrm{H}(4) & 0.6813 & 0.7071 & 0.4391 & 0.098(7) \\ \mathrm{H}(5) & 0.5375 & 0.4817 & 0.4964 & 0.098(7) \\ \mathrm{H}(6) & 0.3971 & 0.3038 & 0.4066 & 0.098(7) \\ \mathrm{H}(8) & 0.2900 & -0.0467 & 0.2709 & 0.098(7) \\ \mathrm{H}(9) & 0.1088 & -0.1954 & 0.3811 & 0.098(7) \\ \mathrm{H}(10) & -0.0941 & -0.0911 & 0.4603 & 0.098(7) \\ \mathrm{H}(11) & -0.1683 & 0.1679 & 0.4384 & 0.098(7) \\ \mathrm{H}(15) & 0.6383 & 0.2242 & 0.2318 & 0.098(7) \\ \mathrm{H}(16) & 0.8075 & 0.0533 & 0.1686 & 0.098(7) \\ \mathrm{H}(17) & 0.7242 & -0.1354 & 0.0359 & 0.098(7) \\ \mathrm{H}(18) & 0.4446 & -0.1613 & -0.0018 & 0.098(7) \\ \mathrm{H}(131) & -0.0421 & 0.4908 & 0.3759 & 0.098(7) \\ \mathrm{H}(132) & -0.1578 & 0.4231 & 0.2770 & 0.098(7) \\ \mathrm{H}(133) & 0.0431 & 0.4751 & 0.2693 & 0.098(7) \\ \mathrm{H}(201) & -0.0179 & -0.0471 & -0.0241 & 0.098(7) \\ \mathrm{H}(202) & 0.1394 & -0.1900 & -0.0019 & 0.098(7) \\ \mathrm{H}(203) & 0.1675 & -0.0372 & -0.0777 & 0.098(7)\end{array}$

Table S13: Hydrogen atoms interatomic distances $(\AA)$ and bond angles $\left(^{\circ}\right)$ for the compound 14

$\begin{array}{lc}\mathrm{C}(2)-\mathrm{H}(2) & 1.058(8) \\ \mathrm{C}(3)-\mathrm{H}(3) & 0.953(9) \\ \mathrm{C}(4)-\mathrm{H}(4) & 1.016(8) \\ \mathrm{C}(6)-\mathrm{H}(6) & 0.892(8) \\ \mathrm{C}(9)-\mathrm{H}(9) & 0.82(1) \\ \mathrm{C}(11)-\mathrm{H}(11) & 1.036(9) \\ \mathrm{C}(13)-\mathrm{H}(132) & 1.031(9) \\ \mathrm{C}(15)-\mathrm{H}(15) & 0.984(7) \\ \mathrm{C}(17)-\mathrm{H}(17) & 0.952(7) \\ \mathrm{C}(20)-\mathrm{H}(201) & 1.07(1) \mathrm{n} \\ \mathrm{C}(20)-\mathrm{H}(203) & 1.02(1) \\ \mathrm{C}(1)-\mathrm{C}(2)-\mathrm{H}(2) & 110.9(8) \\ \mathrm{C}(2)-\mathrm{C}(3)-\mathrm{H}(3) & 112.2(10) \\ \mathrm{C}(3)-\mathrm{C}(4)-\mathrm{H}(4) & 126.0(10) \\ \mathrm{C}(4)-\mathrm{C}(5)-\mathrm{H}(5) & 124.1(10) \\ \mathrm{C}(1)-\mathrm{C}(6)-\mathrm{H}(6) & 126.0(8) \\ \mathrm{C}(7)-\mathrm{C}(8)-\mathrm{H}(8) & 114.7(8) \\ \mathrm{C}(8)-\mathrm{C}(9)-\mathrm{H}(9) & 131.1(12) \\ \mathrm{C}(9)-\mathrm{C}(10)-\mathrm{H}(10) & 121.3(13) \\ \mathrm{C}(10)-\mathrm{C}(11)-\mathrm{H}(11) & 102.9(11) \\ \mathrm{C}(12)-\mathrm{C}(13)-\mathrm{H}(131) & 112.8(9) \\ \mathrm{H}(131)-\mathrm{C}(13)-\mathrm{H}(132) & 107.1(8) \\ \mathrm{H}(131)-\mathrm{C}(13)-\mathrm{H}(133) & 110.7(10) \\ \mathrm{C}(14)-\mathrm{C}(15)-\mathrm{H}(15) & 119.8(8) \\ \mathrm{C}(15)-\mathrm{C}(16)-\mathrm{H}(16) & 107.8(8) \\ \mathrm{C}(16)-\mathrm{C}(17)-\mathrm{H}(17) & 121.4(8) \\ \mathrm{C}(17)-\mathrm{C}(18)-\mathrm{H}(18) & 115.2(9) \\ \mathrm{O}(1)-\mathrm{C}(20)-\mathrm{H}(201) & 108.9(9) \\ \mathrm{H}(201)-\mathrm{C}(20)-\mathrm{H}(202) & 109.4(11) \\ \mathrm{H}(201)-\mathrm{C}(20)-\mathrm{H}(203) & 107.3(12)\end{array}$

$\begin{array}{ll}\mathrm{C}(5)-\mathrm{H}(5) & 0.907(9) \\ \mathrm{C}(8)-\mathrm{H}(8) & 0.985(8) \\ \mathrm{C}(10)-\mathrm{H}(10) & 1.025(9) \\ \mathrm{C}(13)-\mathrm{H}(131) & 1.06(1) \\ \mathrm{C}(13)-\mathrm{H}(133) & 0.98(1) \\ \mathrm{C}(16)-\mathrm{H}(16) & 0.877(7) \\ \mathrm{C}(18)-\mathrm{H}(18) & 0.930(8) \\ \mathrm{C}(20)-\mathrm{H}(202) & 0.99(2)\end{array}$

$\mathrm{C}(3)-\mathrm{C}(2)-\mathrm{H}(2)$

$128.6(8)$

$\mathrm{C}(4)-\mathrm{C}(3)-\mathrm{H}(3) \quad 126.4(10)$

$\mathrm{C}(5)-\mathrm{C}(4)-\mathrm{H}(4) \quad 112.0(10)$

$\mathrm{C}(6)-\mathrm{C}(5)-\mathrm{H}(5) \quad 115.6(10)$

$\mathrm{C}(5)-\mathrm{C}(6)-\mathrm{H}(6)$

$\mathrm{C}(9)-\mathrm{C}(8)-\mathrm{H}(8) \quad 123.7(9)$

$\mathrm{C}(10)-\mathrm{C}(9)-\mathrm{H}(9) \quad 108.2(11)$

$\mathrm{C}(11)-\mathrm{C}(10)-\mathrm{H}(10) \quad 119.8(12)$

$\mathrm{C}(12)-\mathrm{C}(11)-\mathrm{H}(11) \quad 135.9(12)$

$\mathrm{C}(12)-\mathrm{C}(13)-\mathrm{H}(132) \quad 108.1(9)$

$\mathrm{C}(12)-\mathrm{C}(13)-\mathrm{H}(133) \quad 109.7(8)$

$\mathrm{H}(132)-\mathrm{C}(13)-\mathrm{H}(133) \quad 108.3(9)$

$\mathrm{C}(16)-\mathrm{C}(15)-\mathrm{H}(15) \quad 118.8(7)$

$\mathrm{C}(17)-\mathrm{C}(16)-\mathrm{H}(16) \quad 131.5(9)$

$\mathrm{C}(18)-\mathrm{C}(17)-\mathrm{H}(17) \quad 118.1(8)$

$\mathrm{C}(19)-\mathrm{C}(18)-\mathrm{H}(18) \quad 123.6(8)$

$\mathrm{O}(1)-\mathrm{C}(20)-\mathrm{H}(202) \quad 111.7(11)$

$\mathrm{O}(1)-\mathrm{C}(20)-\mathrm{H}(203) \quad 110.7(10)$

$\mathrm{H}(202)-\mathrm{C}(20)-\mathrm{H}(203) \quad 108.7(10)$ 
Structure and crystal data for the $(S, S)-(-)-1,2-b i s(m e t h y l p h e n y l p h o s p h i n i t o$ borane) benzene 18 .

Figure S3

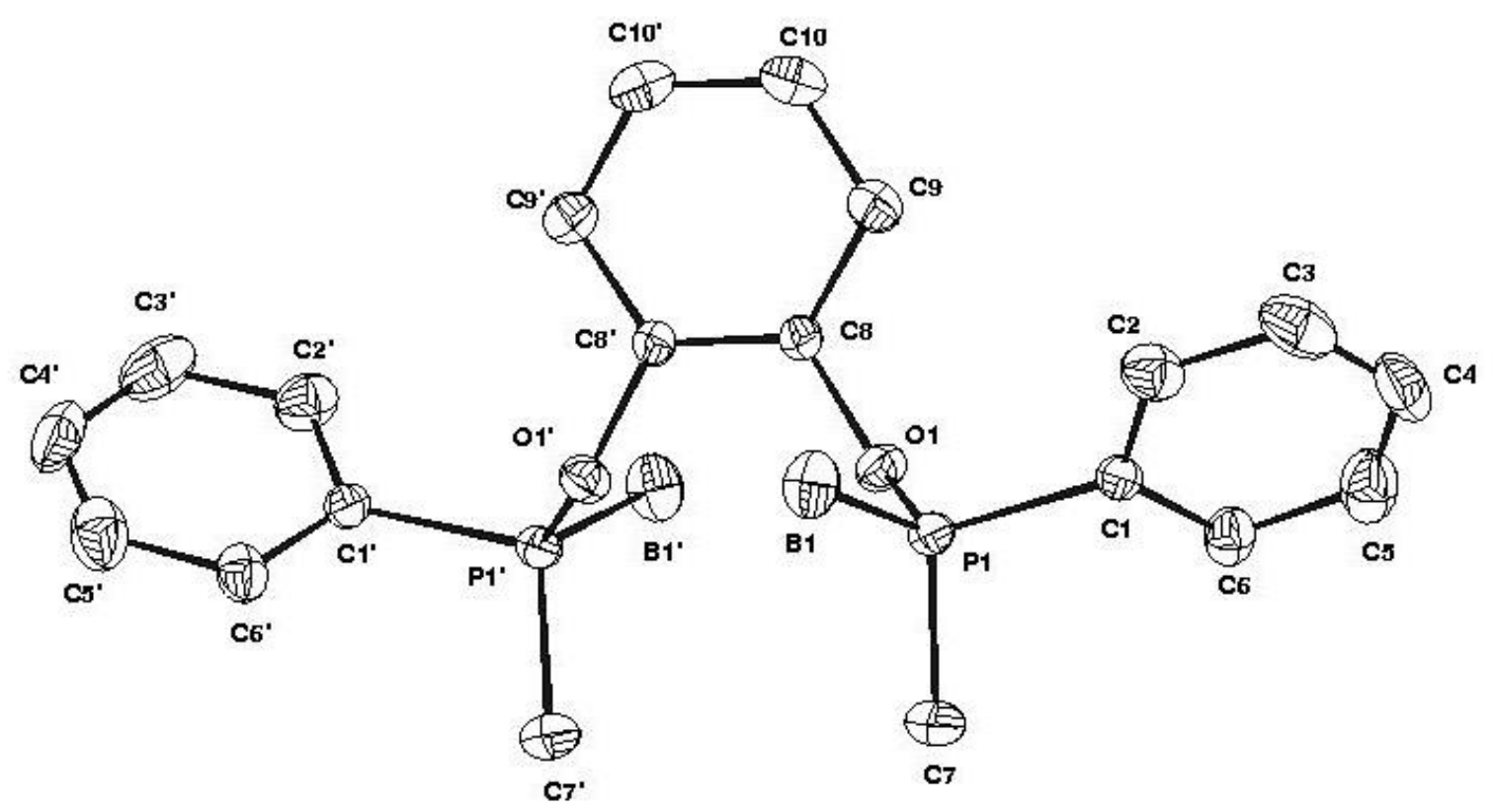

Table S14 CRYSTAL DATA

Formula

Fw =

Crystal System

a $(\underset{\AA}{\mathrm{A}})=$

b $(\AA)=$

c $(\AA)=$

$\alpha($ deg. $)=$

$\beta$ (deg.) $=$

$\gamma($ deg. $)=$

$\mathbf{V}\left(\AA^{3}\right)=$

$\mathrm{Z}=$

Space group

Crystal shape

Crystal colour

Linear absorption coefficient $\mu\left(\mathrm{cm}^{-1}\right)$

Density $\rho\left(\mathrm{g} \mathrm{cm}^{3}\right)$

Diffractometer

Radiation

Scan type

Scan range $\left({ }^{\circ}\right)$

$\theta$ Limits $\left({ }^{\circ}\right)$

Temperature of measurement

Octants collected

$\mathrm{Nb}$ of data collected

$\mathrm{Nb}$ of unique data collected

$\mathrm{Nb}$ of unique data used for refinement
$\mathrm{C}_{20} \mathrm{H}_{26} \mathrm{~B}_{2} \mathrm{O}_{2} \mathrm{P}_{2}$

382.00

Tetragonal

6.830 (2)

6.830 (3)

46.829 (14)

90.

90.

90.

2185 (1)

4

P 41212

Parallelepiped

colorless

4.08

1.16

MACH 3-Enraf Nonius

$\operatorname{MoK} \alpha(\lambda=0.71069 \AA)$

$\omega / 2 \theta$

$0.8+0.345 \operatorname{tg} \theta$

1 - 25

Room temperature

0,$8 ; 0,8 ; 0,55$

2318

1253

$776(\mathrm{Fo})^{2}>2 \sigma(\mathrm{Fo})^{2}$ 
Merging R

Decay of standards reflections \%

$\mathbf{R}=\Sigma|| \mathbf{F o}-|\mathbf{F c} \| / \Sigma| \mathbf{F o} \mid$

$\mathrm{Rw}^{*}=\left[\Sigma \mathrm{w}(\mathrm{Fo}-|\mathbf{F c}|)^{2} / \Sigma \mathbf{w F o}^{2}\right]^{1 / 2}$

Secondary extinction coefficient

Goodness of fit

Nb of variables

$\Delta \rho \min \left(\mathrm{e} / \AA^{3}\right)$

$\Delta \rho \max \left(\mathrm{e}^{\mathrm{A}} \mathbf{3}\right)$
9.9

0.082

0.087

none

0.945

119

$-0.41$

0.42

* Weighting scheme of the form $w=w^{\prime}\left[1-((|| F o|-| F c||) / 6 \sigma(F o))^{2}\right]^{2}$ with $w^{\prime}=1 / \Sigma_{r} A_{r} T_{r}(X)$ with coefficients 4.70, 1.94 and 3.47 for a Chebyshev serie for which $\mathrm{X}=\mathrm{Fc} / \mathrm{Fc}(\mathrm{max})$

Table S15 : Anisotropic thermal parameters for $\mathrm{C}_{20} \mathrm{H}_{26} \mathrm{~B}_{2} \mathrm{O}_{2} \mathrm{P}_{2}$

\begin{tabular}{llllrrr} 
Atom & $\mathrm{U}(11)$ & $\mathrm{U}(22)$ & $\mathrm{U}(33)$ & $\mathrm{U}(23)$ & $\mathrm{U}(13)$ & $\mathrm{U}(12)$ \\
$\mathrm{P}(1)$ & $0.043(2)$ & $0.052(2)$ & $0.049(2)$ & $0.005(1)$ & $-0.006(1)$ & \multicolumn{1}{c}{$-0.008(1)$} \\
$\mathrm{B}(1)$ & $0.06(1)$ & $0.09(1)$ & $0.065(9)$ & $-0.008(9)$ & $0.011(8)$ & $0.02(1)$ \\
$\mathrm{O}(1)$ & $0.048(5)$ & $0.062(5)$ & $0.047(4)$ & $0.000(4)$ & $-0.004(4)$ & $-0.007(4)$ \\
$\mathrm{C}(1)$ & $0.058(8)$ & $0.048(7)$ & $0.039(6)$ & $0.005(6)$ & $-0.011(5)$ & $-0.010(7)$ \\
$\mathrm{C}(2)$ & $0.09(1)$ & $0.08(1)$ & $0.058(7)$ & $0.006(8)$ & $0.006(8)$ & $-0.013(9)$ \\
C(3) & $0.14(2)$ & $0.09(1)$ & $0.08(1)$ & $0.021(9)$ & $0.03(1)$ & $-0.02(1)$ \\
$\mathrm{C}(4)$ & $0.17(2)$ & $0.06(1)$ & $0.08(1)$ & $0.015(9)$ & $-0.05(1)$ & $-0.01(1)$ \\
$\mathrm{C}(5)$ & $0.11(1)$ & $0.08(1)$ & $0.09(1)$ & $-0.01(1)$ & $-0.02(1)$ & $0.02(1)$ \\
$\mathrm{C}(6)$ & $0.07(1)$ & $0.060(9)$ & $0.058(7)$ & $-0.011(7)$ & $-0.015(7)$ & $0.008(7)$ \\
$\mathrm{C}(7)$ & $0.071(9)$ & $0.07(1)$ & $0.094(9)$ & $0.018(8)$ & $-0.009(8)$ & $-0.025(7)$ \\
$\mathrm{C}(8)$ & $0.043(7)$ & $0.051(7)$ & $0.050(7)$ & $0.012(5)$ & $-0.004(5)$ & $-0.003(6)$ \\
C(9) & $0.06(1)$ & $0.053(8)$ & $0.062(7)$ & $-0.000(7)$ & $0.004(7)$ & $-0.000(6)$ \\
C(10) & $0.09(1)$ & $0.058(9)$ & $0.07(1)$ & $-0.000(6)$ & $0.011(8)$ & $-0.020(8)$
\end{tabular}

Table S16: Hydrogen atoms fractional atomic coordinates for $\mathrm{C}_{20} \mathrm{H}_{26} \mathrm{~B}_{2} \mathrm{O}_{2} \mathrm{P}_{2}$

$\begin{array}{lcccc}\text { Atom } & \mathrm{x} / \mathrm{a} & \mathrm{y} / \mathrm{b} & \mathrm{z} / \mathrm{c} & \mathrm{U}(\mathrm{iso}) \\ \mathrm{H}(21) & 0.1917 & 0.3769 & -0.0957 & 0.10(2) \\ \mathrm{H}(31) & 0.0628 & 0.6464 & -0.1266 & 0.10(2) \\ \mathrm{H}(41) & -0.2541 & 0.7574 & -0.1210 & 0.10(2) \\ \mathrm{H}(51) & -0.4574 & 0.6230 & -0.0868 & 0.10(2) \\ \mathrm{H}(61) & -0.3535 & 0.3614 & -0.0553 & 0.10(2) \\ \mathrm{H}(71) & -0.2909 & 0.0822 & -0.0344 & 0.10(2) \\ \mathrm{H}(72) & -0.2218 & -0.0612 & -0.0604 & 0.10(2) \\ \mathrm{H}(73) & -0.1404 & -0.0985 & -0.0281 & 0.10(2) \\ \mathrm{H}(91) & 0.2109 & 0.6051 & -0.0329 & 0.10(2) \\ \mathrm{H}(101) & 0.5308 & 0.7094 & -0.0170 & 0.10(2)\end{array}$


S18

Table S17 : Fractional atomic coordinates for $\mathrm{C}_{20} \mathrm{H}_{26} \mathrm{~B}_{2} \mathrm{O}_{2} \mathrm{P}_{2}$

$\begin{array}{lcccc}\text { Atom } & \mathrm{x} / \mathrm{a} & \mathrm{y} / \mathrm{b} & \mathrm{z} / \mathrm{C} & \mathrm{U}(\mathrm{eqv}) \\ \mathrm{P}(1) & 0.0259(5) & 0.1638(5) & -0.04942(6) & 0.0469 \\ \mathrm{~B}(1) & 0.255(3) & 0.038(3) & -0.0643(3) & 0.0675 \\ \mathrm{O}(1) & 0.048(1) & 0.269(1) & -0.0182(2) & 0.0518 \\ \mathrm{C}(1) & -0.071(2) & 0.353(2) & -0.0723(2) & 0.0460 \\ \mathrm{C}(2) & 0.050(2) & 0.431(2) & -0.0928(3) & 0.0734 \\ \mathrm{C}(3) & -0.022(3) & 0.578(3) & -0.1110(3) & 0.0903 \\ \mathrm{C}(4) & -0.208(3) & 0.642(2) & -0.1074(4) & 0.0827 \\ \mathrm{C}(5) & -0.321(3) & 0.568(3) & -0.0874(4) & 0.0885 \\ \mathrm{C}(6) & -0.258(2) & 0.421(2) & -0.0701(3) & 0.0608 \\ \mathrm{C}(7) & -0.172(2) & 0.002(2) & -0.0422(3) & 0.0720 \\ \mathrm{C}(8) & 0.231(2) & 0.339(2) & -0.0093(2) & 0.0469 \\ \mathrm{C}(9) & 0.294(2) & 0.519(2) & -0.0190(3) & 0.0596 \\ \text { C (10) } & 0.474(2) & 0.584(2) & -0.0097(3) & 0.0682\end{array}$

Table S18 : Interatomic distances ( ) for $\mathrm{C}_{20} \mathrm{H}_{26}{ }_{6} \mathrm{~B}_{2} \mathrm{O}_{2} \mathrm{P}_{2}$

$\begin{array}{llllll}\mathrm{P}(1) & -\mathrm{B}(1) & 1.92(2) & \mathrm{P}(1)-\mathrm{O}(1) & 1.636(8) \\ \mathrm{P}(1)-\mathrm{C}(1) & 1.80(1) & \mathrm{P}(1)-\mathrm{C}(7) & 1.78(1) \\ \mathrm{O}(1)-\mathrm{C}(8) & 1.40(1) & \mathrm{C}(1)-\mathrm{C}(2) & 1.37(2) \\ \mathrm{C}(1)-\mathrm{C}(6) & 1.36(2) & \mathrm{C}(2)-\mathrm{C}(3) & 1.40(2) \\ \mathrm{C}(3)-\mathrm{C}(4) & 1.35(3) & \mathrm{C}(4)-\mathrm{C}(5) & 1.31(2) \\ \mathrm{C}(5)-\mathrm{C}(6) & 1.36(2) & \mathrm{C}(8)-\mathrm{C}(8), & 1.35(2) \\ \mathrm{C}(8)-\mathrm{C}(9) & 1.38(2) & \mathrm{C}(9)-\mathrm{C}(10) & 1.37(2) \\ \mathrm{C}(10)-\mathrm{C}(10)^{\prime} & 1.40(3) & & & \end{array}$

Table S19 : Bond angles (i) for $\mathrm{C}_{20} \mathrm{H}_{26}{ } \mathrm{~B}_{2} \mathrm{O}_{2} \mathrm{P}_{2}$

$\begin{array}{lllllllr}\mathrm{B}(1) & -\mathrm{P}(1) & -\mathrm{O}(1) & 116.5(6) & \mathrm{B}(1) & -\mathrm{P}(1) & -\mathrm{C}(1) & 113.8(7) \\ \mathrm{O}(1) & -\mathrm{P}(1) & -\mathrm{C}(1) & 104.6(5) & \mathrm{B}(1) & -\mathrm{P}(1) & -\mathrm{C}(7) & 114.3(8) \\ \mathrm{O}(1) & -\mathrm{P}(1) & -\mathrm{C}(7) & 99.9(6) & \mathrm{C}(1) & -\mathrm{P}(1) & -\mathrm{C}(7) & 106.2(7) \\ \mathrm{P}(1) & -\mathrm{O}(1) & -\mathrm{C}(8) & 119.9(7) & \mathrm{P}(1) & -\mathrm{C}(1) & -\mathrm{C}(2) & 118.4(12) \\ \mathrm{P}(1) & -\mathrm{C}(1) & -\mathrm{C}(6) & 122.8(10) & \mathrm{C}(2) & -\mathrm{C}(1) & -\mathrm{C}(6) & 118.8(13) \\ \mathrm{C}(1) & -\mathrm{C}(2) & -\mathrm{C}(3) & 119.6(16) & \mathrm{C}(2) & -\mathrm{C}(3) & -\mathrm{C}(4) & 119.0(15) \\ \mathrm{C}(3) & -\mathrm{C}(4) & -\mathrm{C}(5) & 120.9(16) & \mathrm{C}(4) & -\mathrm{C}(5) & -\mathrm{C}(6) & 121.5(17) \\ \mathrm{C}(1) & -\mathrm{C}(6) & -\mathrm{C}(5) & 120.2(15) & \mathrm{O}(1) & -\mathrm{C}(8) & -\mathrm{C}(8), 119.3(6) \\ \mathrm{O}(1) & -\mathrm{C}(8) & -\mathrm{C}(9) & 119.0(10) & \mathrm{C}(8), & -\mathrm{C}(8) & -\mathrm{C}(9) & 121.7(7) \\ \mathrm{C}(8) & -\mathrm{C}(9) & -\mathrm{C}(10) 117.4(12) & \mathrm{C}(9) & -\mathrm{C}(10) & -\mathrm{C}(10) & 120.9(8)\end{array}$

Symmetry code : $\mathrm{y}, \mathrm{x},-\mathrm{z}$ 
Crystal structure and data of the $(+)-N, N^{\prime}-b i s[(R)-o$-anisylphenylphosphino borane] ethylenediamine 20 .

Figure $\mathbf{S 4}$

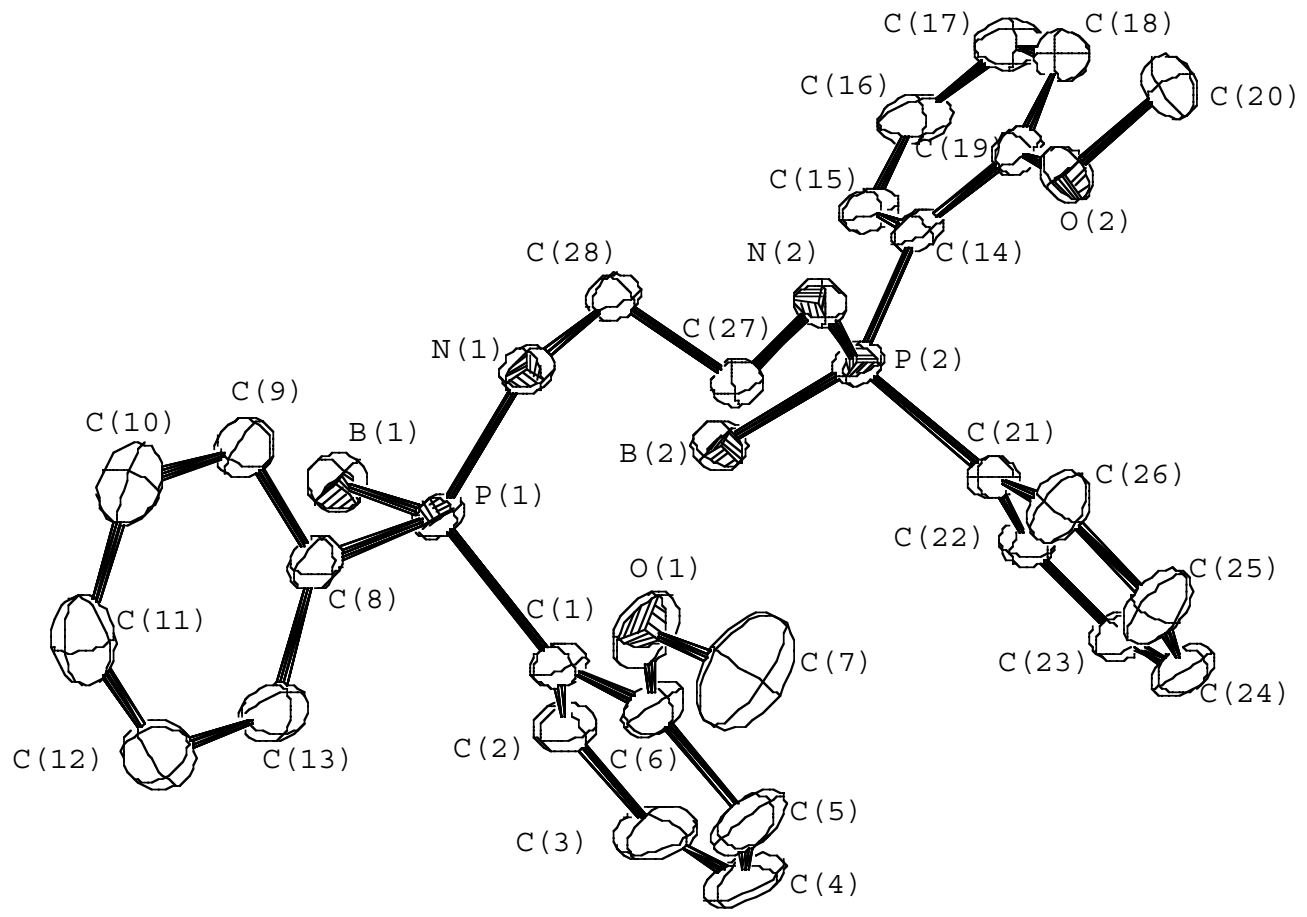

Table S20:

Formula: $\mathrm{C}_{28} \mathrm{H}_{36} \mathrm{~B}_{2} \mathrm{~N}_{2} \mathrm{O}_{2} \mathrm{P}_{2}$; M: 516.15; T, K: 110(2)

Crystal system: Orthorhombic

Space group $\mathrm{P} 21212$
a: $\quad \AA 8.7003(2)$
b: $\quad \AA 12.3994(4)$
c: $\quad \AA 27.423(1)$
$\mathrm{V}: \quad \AA^{3} 2958.35(16)$
$\mathrm{Z}: \quad 4$

$\mathrm{F}(000): \quad 1096$

$D_{\text {calc: }}: \mathrm{g} / \mathrm{cm}^{3} \quad 1.159$

Diffractometer: $\quad$ Enraf-Nonius KappaCCD

Scan type: $\quad$ mixture of $\Phi$ rotations and $\omega$ scans

$\lambda: \AA \quad 0.71073$

$\mu: \mathrm{mm}^{-1} \quad 0.173$

Crystal size: $\mathrm{mm}^{3} \quad 0.25 \times 0.15 \times 0.08$

$\sin (\theta) / \lambda \max : \AA^{-1} \quad 0.65$

Index ranges $\mathrm{h}:-11 ; 7$

$\mathrm{k}:-15 ; 16$

l: $-24 ; 35$

Absorption correction

$\mathrm{RC}=$ Refl. Collected SCALEPACK

IRC $=$ independent RC $\quad 6279$ [R(int $)=0.0646]$

IRCGT $=$ IRC and $[\mathrm{I}>2 \sigma(\mathrm{I})] 5060$

Refinement method: Full-matrix least-squares on $\mathrm{F}^{\wedge} 2$ 
Data / restraints / parameters 6279 / 0 / 333

$\mathrm{R}$ for IRCGT $\mathrm{R} 1^{\mathrm{a}}=0.0548, \mathrm{wR} 2^{\mathrm{b}}=0.1162$

$\mathrm{R}$ for IRC $\quad \mathrm{R} 1^{\mathrm{a}}=0.0764, \mathrm{wR} 2^{\mathrm{b}}=0.1272$

Goodness-of-fit ${ }^{\mathrm{c}} \quad 1.044$

Largest diff. peak and hole; e. $\AA^{-3} \quad 0.294$ and -0.268

a $\mathrm{R} 1=\Sigma($ IIFol- IFcll $) / \Sigma$ IFol.

$\mathrm{b}_{\mathrm{wR} 2}=\left[\Sigma \mathrm{w}\left(\mathrm{Fo}^{2}-\mathrm{Fc}^{2}\right)^{2} / \Sigma\left[\mathrm{w}\left(\mathrm{Fo}^{2}\right)^{2}\right]^{1 / 2}\right.$ where $\mathrm{w}=1 /\left[\sigma^{2}\left(\mathrm{Fo}^{2}\right)+1.06 * \mathrm{P}+(0.0470 * \mathrm{P})^{2}\right]$ where $\mathrm{P}=(\mathrm{Max}$ $\left.\left(\mathrm{Fo}^{2}, 0\right)+2 * \mathrm{Fc}^{2}\right) / 3$

$\mathrm{c}$ Goodness of fit $=\left[\Sigma \mathrm{W}\left(\mathrm{Fo}^{2}-\mathrm{Fc}^{2}\right)^{2} /\left(\mathrm{N}_{\mathrm{o}}-\mathrm{N}_{\mathrm{v}}\right)\right]^{1 / 2}$.

Table S21:

Atomic coordinates $\left(\mathrm{x} 10^{\wedge} 4\right)$ and equivalent isotropic displacement parameters $\left(\mathrm{A}^{\wedge} 2 \times 10^{\wedge} 3\right)$ for:

(compound 20)

$\begin{array}{llll} & \mathrm{x} & \mathrm{y} & \mathrm{z} \\ \mathrm{P}(1) & 4372(1) & 4251(1) & 8257(1) \\ \mathrm{P}(2) & 3209(1) & 4307(1) & 6253(1) \\ \mathrm{B}(1) & 5043(4) & 5696(3) & 8141(1) \\ \mathrm{B}(2) & 2982(4) & 5718(3) & 6527(2) \\ \mathrm{N}(1) & 5349(2) & 3357(2) & 7928(1) \\ \mathrm{N}(2) & 4241(2) & 3481(2) & 6596(1) \\ 0(1) & 2614(2) & 2250(2) & 8357(1) \\ 0(2) & 4158(2) & 2430(2) & 5660(1) \\ \mathrm{C}(27) & 4003(3) & 3424(3) & 7125(1) \\ \mathrm{C}(28) & 5513(3) & 3532(3) & 7399(1)\end{array}$

Table S22: Bond lengths $[\AA ̊]$ and angles [deg] for: (compound 20)

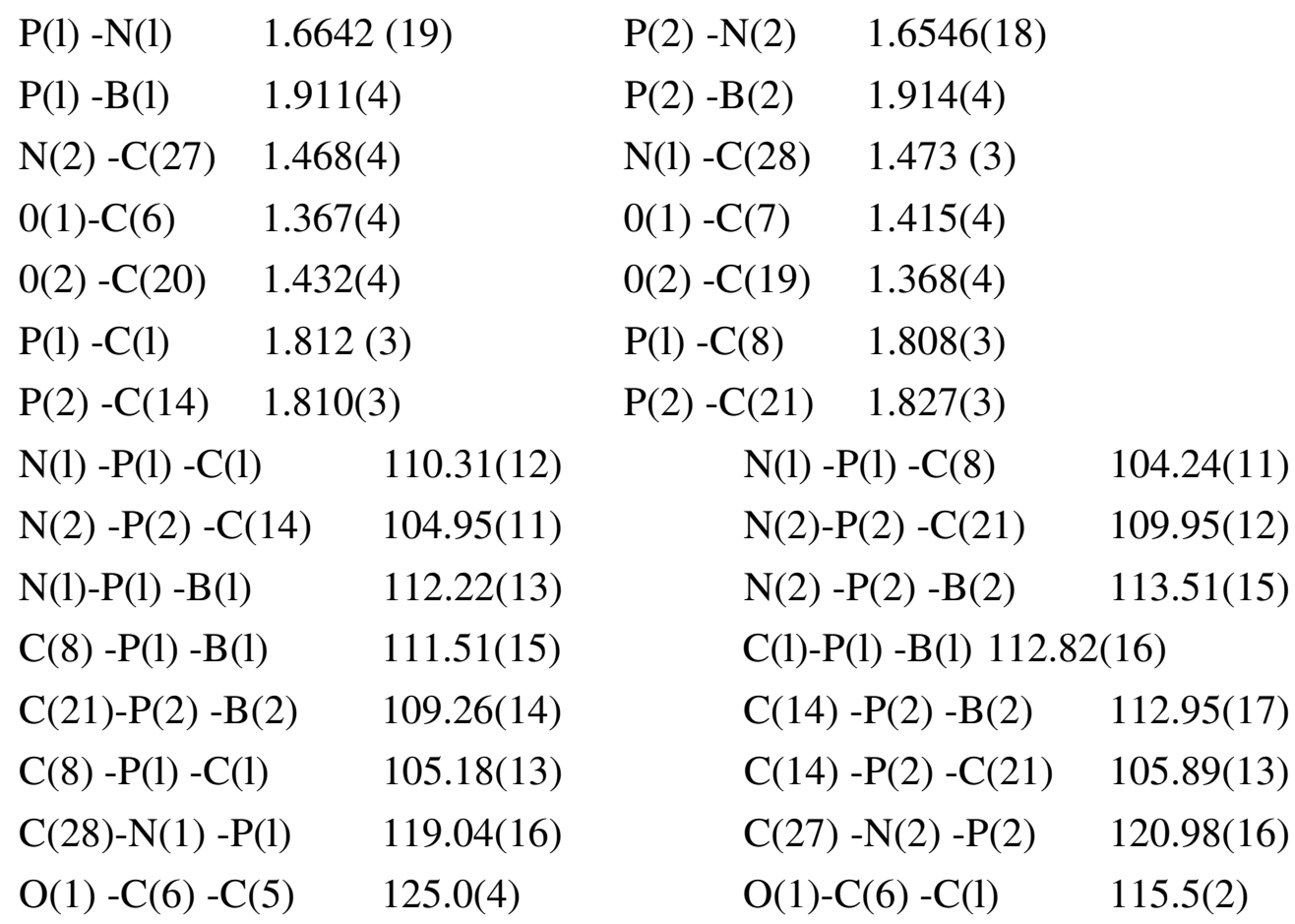


$\mathrm{O}(2)-\mathrm{C}(19)-\mathrm{C}(18) \quad 123.8(3)$

$\mathrm{C}(6)-\mathrm{O}(1)-\mathrm{C}(7) \quad 118.8(3)$

$\mathrm{C}(9)-\mathrm{C}(8)-\mathrm{P}(1) \quad 120.0(2)$

$\mathrm{C}(2)-\mathrm{C}(1)-\mathrm{P}(1) \quad 119.3(3)$

$\mathrm{C}(15)-\mathrm{C}(14)-\mathrm{P}(2) \quad 119.2(2)$

$\mathrm{C}(22)-\mathrm{C}(21)-\mathrm{P}(2) \quad 119.7(2)$

$\mathrm{N}(1)-\mathrm{H} . . \mathrm{H}-\mathrm{B}(1) \quad 2.27 \AA$

$\mathrm{N}(2)-\mathrm{H} . . \mathrm{H}-\mathrm{B}(2) \quad 2.26 \AA$

$\mathrm{N}(1)-\mathrm{H} . . \mathrm{O}(1) \mathrm{C}(7) \quad 2.43 \AA$

$\mathrm{N}(2)-\mathrm{H} . . \mathrm{O}(2) \mathrm{C}(20) \quad 2.31 \AA$

Diedral angles $\left(^{\circ}\right)$

$\mathrm{N}(2)-\mathrm{P}(2)-\mathrm{C}(21)-\mathrm{C}(26) \quad 1.5$

$\mathrm{P}(1)-\mathrm{B}(1)-\mathrm{P}(2)-\mathrm{B}(2) \quad 30.1$

$\mathrm{P}(1)-\mathrm{C}(1)-\mathrm{C}(6)-\mathrm{O}(1) \quad 1$

$\mathrm{C}(1)-\mathrm{C}(6)-\mathrm{O}(1)-\mathrm{C}(7) \quad 179$

$\mathrm{B}(1)-\mathrm{P}(1)-\mathrm{C}(1)-\mathrm{C}(6) \quad 178$

$\mathrm{N}(1)-\mathrm{P}(1)-\mathrm{C}(8)-\mathrm{C}(9) \quad 36.2$

$\mathrm{C}(13)-\mathrm{C}(8)-\mathrm{P}(1)-\mathrm{C}(1) \quad 32.8$

$\mathrm{N}(1)-\mathrm{C}(28)-\mathrm{C}(27)-\mathrm{N}(2) \quad 173.5$

$\mathrm{N}(1)-\mathrm{P}(1)-\mathrm{N}(2)-\mathrm{P}(2) \quad 10$

$\mathrm{P}(2)-\mathrm{C}(14)-\mathrm{C}(19)-\mathrm{O}(2) \quad 2.7$

$\mathrm{C}(14)-\mathrm{C}(19)-\mathrm{O}(2)-\mathrm{C}(20) \quad 176.5$

$\mathrm{B}(2)-\mathrm{P}(2)-\mathrm{C}(14)-\mathrm{C}(19) \quad 176.3$
$\mathrm{O}(2)-\mathrm{C}(19)-\mathrm{C}(14) \quad 115.3\{3)$

$\mathrm{C}(19)-\mathrm{O}(2)-\mathrm{C}(20) \quad 118.4(2)$

$\mathrm{C}(13)-\mathrm{C}(8)-\mathrm{P}(\mathrm{l}) \quad 121.1(2)$

$\mathrm{C}(6)-\mathrm{C}(1)-\mathrm{P}(\mathrm{l}) \quad 120.6(2)$

$\mathrm{C}(19)-\mathrm{C}(14)-\mathrm{P}(2) \quad 121.9(2)$

$\mathrm{C}(26)-\mathrm{C}(21)-\mathrm{P}(2) \quad 121.3$ (2) 